\title{
Alkene Substituents for Selective Activation of Endo-Regioselective Polyepoxide Oxacyclizations
}

Fernando Bravo, Frank E. McDonald,* Wade A. Neiwert, and Kenneth I. Hardcastle

Department of Chemistry, Emory University, Atlanta, Georgia 30322

\section{SUPPORTING INFORMATION}

\section{Table of Contents:}

General:

Synthesis of pentaepoxide substrate (4):

Silylation of geraniol and allylic oxidation

S-3

Conversion of allylic alcohol to allylic bromide

S-4

Barium cross-coupling with farnesyl chloride

S-5

Preparation of monoepoxide

S-6

Shi epoxidation to provide pentaepoxide (4)

S-7

Polycyclization of $\mathbf{4}$ to pentacyclic (8)

S-8

Synthesis of $O$-Boc-geraniol-derived monoepoxide (11)

S-9

Synthesis of $O$-Boc-farnesol-derived monoepoxide (12)

S-10

Allylic oxidation of $\mathbf{1 1}$ to produce (13)

S-10

Allylic oxidation of $\mathbf{1 2}$ to produce (14)

S-11

Oxidation and methylenation of $\mathbf{1 3}$

S-12

Shi epoxidation to produce diepoxide (15)

S-13

Synthesis of triepoxide substrate (16):

Sharpless epoxidation of $\mathbf{1 4}$

S-14

Shi epoxidation

S-15

Oxidation and methylenation to provide triepoxide (16)

S-16

Bicyclization of $\mathbf{1 5}$ to (17)

S-17

Crystal structure data for ent-17

S-18 - 24

Thermal ellipsoid diagram for ent-17

$\mathrm{S}-25$

Tricyclization of 16 to (18)

S-26

Bromonium-induced cyclization of 11 to (19) and (20)

S-27

Crystal structure data for $\mathbf{1 9}$

S-28 - 34

Thermal ellipsoid diagram for $\mathbf{1 9}$

S-35 
General: ${ }^{1} \mathrm{H}$ NMR spectra were recorded at either $300 \mathrm{MHz}$ on a Varian Mercury-300, at 400 $\mathrm{MHz}$ on an Inova-400 spectrometer, or at $600 \mathrm{MHz}$ on an INOVA-600 spectrometer. ${ }^{13} \mathrm{C}$ NMR spectra were recorded at $75 \mathrm{MHz}, 100 \mathrm{MHz}$, or $150 \mathrm{MHz}$ on the same instruments. NMR spectra were recorded in deuterated chloroform $\left(\mathrm{CDCl}_{3}\right)$ solutions, with residual chloroform $(\delta$ $7.26 \mathrm{ppm}$ for ${ }^{1} \mathrm{H}$ NMR and $\delta 77.23 \mathrm{ppm}$ for ${ }^{13} \mathrm{C}$ NMR) taken as the internal standard, and were reported in parts per million (ppm). Abbreviations for signal coupling are as follows: s, singlet; d, doublet; t, triplet; q, quartet; m, multiplet. IR spectra were collected on a Mattson Genesis II FT-IR spectrometer as neat films. Elemental analyses were performed by Atlantic Microlab Inc, P. O. Box 2288, Norcross, Georgia. Analytical Thin Layer Chromatography (TLC) was performed on precoated glass backed plates purchased from Whatman (silica gel $60 \mathrm{~F}_{254}$; $0.25 \mathrm{~mm}$ thickness). Flash column chromatography was carried out with silica gel 60 (230-400 mesh ASTM) from EM Science. Optical rotations were recorded with a Perkin-Elmer $241 \mathrm{MC}$ polarimeter.

All reactions were carried out with anhydrous solvents in oven-dried or flame-dried and nitrogen- or argon-charged glassware. All anhydrous solvents except as mentioned were freshly distilled. All solvents used in workup, extraction procedures and chromatography were used as received from commercial suppliers without prior purification. During reaction workup, the reaction mixture was usually diluted to three times the original volume, and washed with equal volume of water and/or aqueous solutions as needed. Purified and redistilled boron trifluoride diethyl etherate $\left(\mathrm{BF}_{3} \mathrm{OEt}_{2}\right)$ was purchased from the Aldrich Chemical Company. All other reagents were purchased from Aldrich Chemical Company. 
$\left(\Delta^{2,3} E, \Delta^{6,7} E\right)-1-O$-tert-butyldiphenylsilyl-3,7-dimethyl-octa-2,6-dien-1,8-diol and $\left(\Delta^{2,3} E\right)-1$ O-tert-butyldiphenylsilyl-3,7-dimethyl-octa-2,6-dien-1,4-diol.

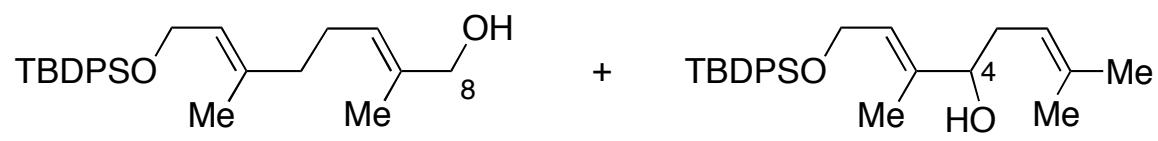

Geraniol (2.67 g, $17.3 \mathrm{mmol})$ was dissolved in anhydrous dichloromethane $(35 \mathrm{~mL})$, and imidazole $(2.59 \mathrm{~g}, 21.3 \mathrm{mmol})$ was added followed by TBDPSCl $(5.00 \mathrm{~mL}, 19.2 \mathrm{mmol})$ in one portion at $0^{\circ} \mathrm{C}$. The reaction was stirred for $1 \mathrm{~h}$, and then it was diluted with dichloromethane, poured over water, and extracted with more dichloromethane. The combination of organic layers was dried over magnesium sulfate, filtered, and the solvent was removed under reduced pressure. The crude thus obtained was purified by column chromatography (pentane:ethyl ether 50:1). The allylic oxidation was performed as follows: in a $250 \mathrm{~mL}$ flask, provided with an addition funnel, $t$-BuOOH $(70 \%$ in water, $4.90 \mathrm{~mL}, 38.1 \mathrm{mmol})$ was added dropwise over a suspension of $\mathrm{SeO}_{2}(190 \mathrm{mg}, 1.71 \mathrm{mmol})$ in dichloromethane $(65 \mathrm{~mL})$. The solution was stirred until it cleared (approx. $10 \mathrm{~min}$.) at room temperature, and then cooled to $0^{\circ} \mathrm{C}$. Then, a solution of the silylated geraniol previously obtained in dichloromethane $(65 \mathrm{~mL})$ was added dropwise through the addition funnel, finishing the addition after $45 \mathrm{~min}$. The reaction was stirred then for $48 \mathrm{~h}$, after which time it was poured over sodium hydroxide (10\% in water) and extracted with $\mathrm{CH}_{2} \mathrm{Cl}_{2}$. The combination of organic phases was washed first with water, then brine. The solvent was dried over $\mathrm{MgSO}_{4}$, the solids were filtered off, and the solvent was removed under reduced pressure. Purification with column chromatography (pentane:ethyl ether, increasing gradually the polarity from $5: 1$ to $3: 1$ to $2: 1)$ afforded the product of oxidation at C-8 position $(1.34 \mathrm{~g}, 19 \%)$ and the product of oxidation at C-4 (685 mg, 10\%), along with $1.46 \mathrm{~g} \mathrm{(21 \% )} \mathrm{of} \mathrm{silylated} \mathrm{geraniol.}$

Product of oxidation at C-4:

${ }^{1} \mathrm{H}$ NMR $\left(400 \mathrm{MHz}, \mathrm{CDCl}_{3}\right) \delta$ 7.80-7.40 (m, 10H), $5.62(\mathrm{tt}, J=6.1,1.3 \mathrm{~Hz}, 1 \mathrm{H}), 5.10(\mathrm{tt}, J=7.2$, $1.4 \mathrm{~Hz}, 1 \mathrm{H}), 4.27(\mathrm{~d}, J=6.4 \mathrm{~Hz}, 1 \mathrm{H}), 3.96(\mathrm{t}, J=6.6 \mathrm{~Hz}, 1 \mathrm{H}), 2.29-2.14(\mathrm{~m}, 2 \mathrm{H}), 1.72(\mathrm{~s}, 3 \mathrm{H})$, $1.64(\mathrm{~s}, 3 \mathrm{H}), 1.45$ (d, $J=1.2 \mathrm{~Hz}, 3 \mathrm{H}), 1.04(\mathrm{~s}, 9 \mathrm{H})$.

${ }^{13} \mathrm{C}$ NMR $(100.5 \mathrm{MHz}) \delta 138.0,135.6,134.9,133.9$, 129.6, 127.6, 125.5, 119.9, 60.8, 33.9, 26.8, 25.9, 19.1, 12.0. 
Product of oxidation at C-8:

IR (neat): 3350 (br), 3071, 3049, 2959, 2931, 2857, 1959, 1889, 1823, 1670, 1589, 1472, 1462 , $1428,1383,1361,1262,1189,1112,1058,1007,939,823,739,702 \mathrm{~cm}^{-1}$.

${ }^{1} \mathrm{H}$ NMR $\left(400 \mathrm{MHz}, \mathrm{CDCl}_{3}\right) \delta$ 7.80-7.40 (m, 10H), 5.42-5.37 (m, 2H), $4.22(\mathrm{dd}, J=6.4,0.4 \mathrm{~Hz}$, $1 \mathrm{H}), 3.89(\mathrm{~s}, 1 \mathrm{H}), 2.13$ (q, $J=7.3 \mathrm{~Hz}, 2 \mathrm{H}), 2.01(\mathrm{t}, J=7.6 \mathrm{~Hz}, 2 \mathrm{H}), 1.67(\mathrm{~s}, 3 \mathrm{H}), 1.45(\mathrm{~s}, 3 \mathrm{H})$, $1.04(\mathrm{~s}, 9 \mathrm{H})$.

${ }^{13} \mathrm{C}$ NMR $(100.5 \mathrm{MHz}) \delta 136.6,135.6,134.9,134.0,129.5,127.6,125.8,124.3,69.0,61.1,39.0$, $26.8,25.8,19.1,16.3,13.7$.

$\left(\Delta^{2,3} E, \Delta^{6,7} E\right)-8$-bromo-1-O-tert-butyldiphenylsilyl-3,7-dimethyl-octa-2,6-dien-1-ol.

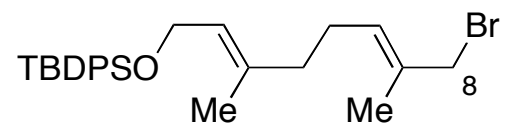

The alcohol product of the oxidation at C-8 previously obtained $(1.34 \mathrm{~g}, 3.29 \mathrm{mmol})$ was dissolved in dichloromethane $(6.5 \mathrm{~mL})$ and triethylamine $(690 \mu \mathrm{L}, 4.95 \mathrm{mmol})$, and the solution was cooled at $-40^{\circ} \mathrm{C}$. Then, mesyl chloride $(300 \mu \mathrm{L}, 3.88 \mathrm{mmol})$ was added dropwise and the reaction was stirred for $1 \mathrm{~h}$ (ATTENTION: the mesylate showed identical $\mathrm{R}_{\mathrm{f}}$ as the alcohol in pentane:ethyl ether 1:1). After that time, the mixture was warmed to $0^{\circ} \mathrm{C}$, and a solution of $\mathrm{LiBr}$ (715 mg, $8.23 \mathrm{mmol})$ in anhydrous THF $(4 \mathrm{~mL})$ was added. After $1 \mathrm{~h}$ at $0^{\circ} \mathrm{C}$, the mixture was poured into water and extracted with $\mathrm{CH}_{2} \mathrm{Cl}_{2}$. The combination of organic layers was dried over magnesium sulfate, filtered, and the solvent was removed under reduced pressure. The crude thus obtained was purified by column chromatography (pentane:ethyl ether, increasing gradually the polarity from 100:1 to 50:1) to afford the title compound (1.34 g, 84\%).

IR (neat): 3071, 3015, 2959, 2931, 2893, 2857, 1959, 1890, 1823, 1667, 1589, 1472, 1428, 1388, $1361,1263,1205,1111,1057,1007,939,823,777,739,702 \mathrm{~cm}^{-1}$.

${ }^{1} \mathrm{H}$ NMR $\left(300 \mathrm{MHz}, \mathrm{CDCl}_{3}\right) \delta 7.80-7.40(\mathrm{~m}, 10 \mathrm{H}), 5.62(\mathrm{t}, J=6.9 \mathrm{~Hz}, 1 \mathrm{H}), 5.30(\mathrm{tt}, J=6.3,1.5$ $\mathrm{Hz}, 1 \mathrm{H}), 4.23$ (d, $J=6.3 \mathrm{~Hz}, 1 \mathrm{H}), 3.96(\mathrm{~s}, 1 \mathrm{H}), 2.13$ (q, $J=7.1 \mathrm{~Hz}, 2 \mathrm{H}), 2.02(\mathrm{t}, J=7.2 \mathrm{~Hz}, 2 \mathrm{H})$, $1.76(\mathrm{~s}, 3 \mathrm{H}), 1.44$ (s, 3H), 1.05 (s, 9H).

${ }^{13} \mathrm{C}$ NMR $(75.4 \mathrm{MHz}) \delta 136.2,135.6,134.0,132.1,130.9,129.5,127.6,124.6,61.0,47.7,38.5$, 26.8, 26.5, 19.1, 16.3, 14.7. 


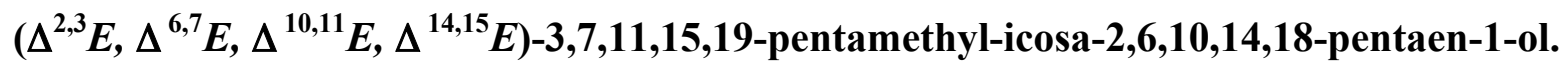

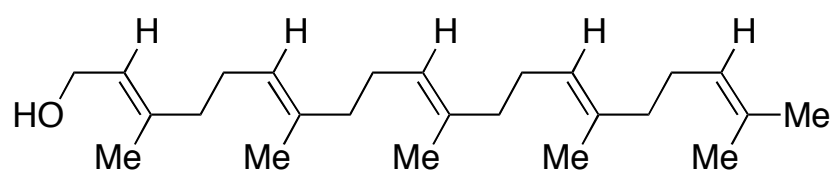

ATTENTION: In order to ensure the reproducibility of the reaction, the following precautions were taken: all glassware used was submitted to three cycles of flame-drying, followed by cooling under argon. The tetrahydrofuran used in the reaction was dried over sodium (benzophenone ketyl) and then thoroughly deoxygenated after three cycles of freezing under vacuum $\left(\mathrm{N}_{2}(1)\right.$ bath) and then melting under argon atmosphere. All the reaction flasks were protected from light. The bromide obtained in the previous reaction was freshly purified prior to use. A solution of biphenyl (165 mg, $1.07 \mathrm{mmol}$, recrystallized from methanol) in THF (2.5 mL) was added over lithium $(7.3 \mathrm{mg}, 1.05 \mathrm{mmol}$ ) and the mixture was stirred for $2 \mathrm{~h}$. After this time, the deep blue-greenish solution of the lithium biphenylide was added dropwise over a slurry of $\mathrm{BaI}_{2}$ (formed from $\mathrm{BaI}_{2} \cdot 2 \mathrm{H}_{2} \mathrm{O}$ after heating it at $200^{\circ} \mathrm{C}$ for $2 \mathrm{~h}, 215 \mathrm{mg}, 0.53 \mathrm{mmol}$ ) in THF $(2.5$ $\mathrm{mL}$ ). The mixture was stirred for $30 \mathrm{~min}$., to form a dark dirty brown suspension of Ba* (Rieke). The flask was then cooled to $-78^{\circ} \mathrm{C}$, and after $5 \mathrm{~min}$. farnesyl chloride $(140 \mu \mathrm{L}, 0.52 \mathrm{mmol})$, dissolved in THF ( $1 \mathrm{~mL})$, was slowly added. The mixture was stirred for $30 \mathrm{~min}$. more, and it turned into a bright red-brick color. Over this generated organobarium reagent, the bromide previously synthesized $(100 \mathrm{mg}, 0.21 \mathrm{mmol})$, dissolved in THF $(1 \mathrm{~mL})$, was added at $-78^{\circ} \mathrm{C}$ and the reaction was allowed to warm up slowly to room temperature. The reaction was quenched after $24 \mathrm{~h}$, by the addition of a few milliliters of $\mathrm{NH}_{4} \mathrm{Cl}$ (aq., sat.), extracted then with ether, and then washed with sodium thiosulfate ( $10 \%$ in water). The combination of organic layers was dried over magnesium sulfate, filtered, and the solvent was removed under reduced pressure. The crude thus obtained was treated with TBAF $3 \mathrm{H}_{2} \mathrm{O}(250 \mathrm{mg}, 0.79 \mathrm{mmol})$ in THF $(1 \mathrm{~mL})$ at $0^{\circ} \mathrm{C}$ for $4 \mathrm{~h}$. The reaction was then poured over water and extracted with dichloromethane. The organic layers were combined, dried over $\mathrm{MgSO}_{4}$, filtered and the solvent was removed under reduced pressure. Purification of the crude by column chromatography (pentane:ethyl ether, increasing gradually the polarity from $10: 1$ to $5: 1$ to $3: 1$ to $1: 1$ ) furnished the title compound (61 $\mathrm{mg}, 83 \%$ yield). This reaction proved to be reproducible up to $500 \mathrm{mg}$ of starting bromide. 
However, a test carried out with $1.0 \mathrm{~g}$ of bromide afforded only a $30 \%$ yield of the desired pentaene.

${ }^{1} \mathrm{H}$ NMR $\left(300 \mathrm{MHz}, \mathrm{CDCl}_{3}\right) \delta 5.42(\mathrm{t}, J=6.9 \mathrm{~Hz}, 1 \mathrm{H}), 5.18-5.02(\mathrm{~m}, 4 \mathrm{H}), 4.15(\mathrm{~d}, J=6.6 \mathrm{~Hz}$, 1H), 2.14-1.92 (m, 16H), 1.62 (app. s, 6H), 1.60 (app. s, 12H).

${ }^{13} \mathrm{C}$ NMR (75.4 MHz) $\delta 135.4,135.0,134.9,132.4,131.3,124.5,124.4,124.2,123.7,123.3$, $59.4,39.7,39.6,32.0,26.8,26.6,26.3,25.7,17.7,16.3,16.0$.

$\left(2 R, 3 R, \Delta^{6,7} E, \Delta^{10,11} E, \Delta^{14,15} E\right)-2,3-$-epoxy-3,7,11,15,19-pentamethyl-icos-6,10,14,18-tetraen-1-ol.

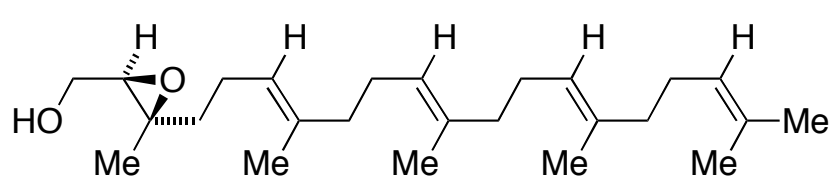

The Sharpless epoxidation was performed as follows: in a $50 \mathrm{~mL}$, round-bottomed flask, $4 \AA$ molecular sieves $(360 \mathrm{mg})$ were dispersed in anhydrous dichloromethane $(10 \mathrm{~mL})$. Then D-(-)diisopropyl tartrate $(30 \mu \mathrm{L}, 0.14 \mathrm{mmol}$ ) was added to the reaction flask and the mixture was cooled to $-40^{\circ} \mathrm{C}$. After $10 \mathrm{~min}$., $\mathrm{Ti}(\mathrm{O}-i-\mathrm{Pr})_{4}(35 \mu \mathrm{L}, 0.12 \mathrm{mmol})$ was added and stirred at $-40^{\circ} \mathrm{C}$ for $15 \mathrm{~min}$. After that time, $t$-BuOOH $(5.0-6.0 \mathrm{M}$ in decane, $320 \mu \mathrm{L} 1.76 \mathrm{mmol})$ was introduced and the mixture was stirred at $-40^{\circ} \mathrm{C}$ for $30 \mathrm{~min}$, after which time the pentaen-ol previously obtained $(425 \mathrm{mg}, 1.19 \mathrm{mmol})$ was added as a solution in dry dichloromethane $(2 \mathrm{~mL})$. The reaction mixture was warmed to $-18^{\circ} \mathrm{C}$ and kept at this temperature overnight. The reaction was quenched by addition of acetone containing $2 \%$ water $(10 \mathrm{~mL})$, warmed at room temperature and stirred for $3 \mathrm{~h}$. After filtering through Celite, the solvent was dried over $\mathrm{MgSO}_{4}$, the solids were filtered, and the solvent evaporated under vacuum. The crude mixture was purified by column chromatography (pentane: ethyl ether from 3:1 to 2:1), to provide the title compound (414 $\mathrm{mg}$, 93\%).

${ }^{1} \mathrm{H}$ NMR (400 MHz, $\left.\mathrm{CDCl}_{3}\right) \delta 5.14-5.02(\mathrm{~m}, 4 \mathrm{H}), 3.82(\mathrm{dd}, J=12.0,4.0 \mathrm{~Hz}, 1 \mathrm{H}), 3.66(\mathrm{dd}, J=$ 12.0, $7.0 \mathrm{~Hz}, 1 \mathrm{H}), 3.29$ (bs, 1H), 2.97 (dd, $J=7.0,4.0 \mathrm{~Hz}, 1 \mathrm{H}), 2.12-2.01$ (m, 10H), 2.00-1.94 (m, 6H), 1.67 (s, 3H), 1.60 (s, 3H), 1.59 (app. s, 9H), 1.29 (s, 3H).

${ }^{13} \mathrm{C}$ NMR $(100.5 \mathrm{MHz}) \delta 135.8,135.0,134.9,131.2,124.3,124.2,124.0,123.1,63.0,61.4,61.2$, $39.7,39.6,38.5,26.7,26.6,26.5,25.7,23.5,21.7,21.6,17.6,16.7,16.0$ (2 carbons). 


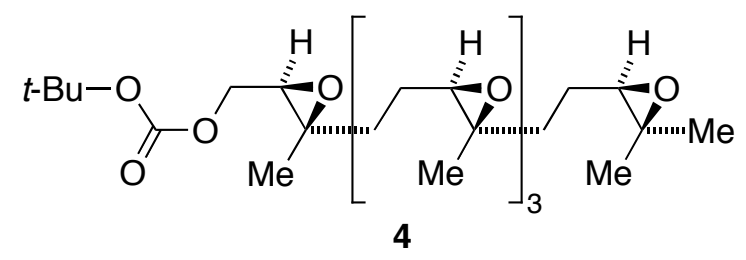

The Shi epoxidation was conducted with the epoxy-alcohol obtained in the previous step (406 $\mathrm{mg}, 1.08 \mathrm{mmol}$ ), which was dissolved in a 1:2 mixture of acetonitrile: dimethoxymethane (15 $\mathrm{mL}$ ). Under vigorous agitation, a $0.05 \mathrm{M}$ solution of $\mathrm{Na}_{2} \mathrm{~B}_{4} \mathrm{O}_{7}$ in $4 \cdot 10^{-4} \mathrm{M} \mathrm{Na}_{2}$ (EDTA) (43 mL), $\mathrm{NBu}_{4} \mathrm{HSO}_{4}(61 \mathrm{mg}, 0.17 \mathrm{mmol}$ ) and Shi catalyst $(336 \mathrm{mg}, 1.30 \mathrm{mmol})$, were sequentially added. The mixture was cooled to $0^{\circ} \mathrm{C}$, and then Oxone $(3.68 \mathrm{~g}, 5.99 \mathrm{mmol})$, dissolved in $4 \cdot 10^{-4} \mathrm{M}$ $\mathrm{Na}_{2}$ (EDTA) $(26 \mathrm{~mL})$, and $\mathrm{K}_{2} \mathrm{CO}_{3}(3.48 \mathrm{~g}, 25.2 \mathrm{mmol})$, dissolved in water $(26 \mathrm{~mL})$, were simultaneously added over the mixture over $1.5 \mathrm{~h}$ period of time. Once the addition was completed, the mixture was stirred for $15 \mathrm{~min}$. more, diluted with water and extracted with dichloromethane. The organic extracts were dried over $\mathrm{MgSO}_{4}$, and the solvent was removed under vacuum. The crude was then dissolved in dichloromethane $(50 \mathrm{~mL})$, and triethylamine (3.3 $\mathrm{mL}, 23.7 \mathrm{mmol}$ ), and $N, N$-dimethylaminopyridine ( $160 \mathrm{mg}, 1.31 \mathrm{mmol}$ ) was added. The solution was cooled to $0^{\circ} \mathrm{C}$ and melted $\mathrm{Boc}_{2} \mathrm{O}(950 \mathrm{mg}, 4.35 \mathrm{mmol})$ was added dropwise. The solution was stirred overnight at room temperature, and then poured over water and extracted with dichloromethane. The organic fractions were combined, dried over magnesium sulfate and the solvent was removed under vacuum. After purification by column chromatography (pentane:ethyl ether 1:3, containing $0.5 \% \mathrm{NEt}_{3}$ ), the title compound 4 was isolated (170 $\mathrm{mg}$, $29 \%)$.

${ }^{1} \mathrm{H}$ NMR $\left(400 \mathrm{MHz}, \mathrm{CDCl}_{3}\right) \delta 4.19(\mathrm{dd}, J=12.0,5.0 \mathrm{~Hz}, 1 \mathrm{H}), 4.12(\mathrm{dd}, J=12.0,6.0 \mathrm{~Hz}, 1 \mathrm{H})$, $3.02(\mathrm{t}, J=5.4 \mathrm{~Hz}, 1 \mathrm{H}), 2.78-2.66(\mathrm{~m}, 4 \mathrm{H}), 1.80-1.40(\mathrm{~m}, 16 \mathrm{H}), 1.47(\mathrm{~s}, 9 \mathrm{H}), 1.32(\mathrm{~s}, 3 \mathrm{H}), 1.29$ (s, 3H), $1.26(\mathrm{~s}, 3 \mathrm{H}), 1.254(\mathrm{~s}, 3 \mathrm{H}), 1.251(\mathrm{~s}, 3 \mathrm{H}), 1.246(\mathrm{~s}, 3 \mathrm{H})$.

${ }^{13} \mathrm{C}$ NMR $(100.5 \mathrm{MHz}) \delta$ 153.2, 82.6, 65.3, 64.1, 63.8, 62.8, 62.6, 62.4, 60.3 (2 carbons), 60.0, 59.0, 58.4, 35.2, 35.1 ( 2 carbons), 34.6, 27.7, 24.8, 24.5, 24.3 (2 carbons), 24.1, 23.8, 22.0, 18.6, $16.9,16.6$. 


\section{Pentacycle (8).}

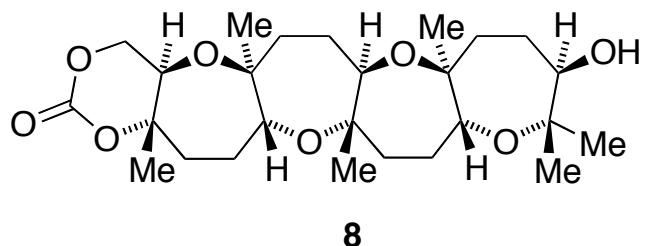

The pentaepoxide 4 (170 mg, $0.32 \mathrm{mmol})$ was dissolved in dichloromethane $(6.3 \mathrm{~mL})$ and the solution was cooled to $-40^{\circ} \mathrm{C}$. Then, a solution of boron trifluoride etherate $(0.2 \mathrm{M}$ in dichloromethane, $1.60 \mathrm{~mL}, 0.32 \mathrm{mmol}$ ) was added dropwise, finishing the addition in $<1 \mathrm{~min}$. The reaction was stirred for $20 \mathrm{~min}$, and then a few milliliters of $\mathrm{NaHCO}_{3}$ (aq., sat.) were added. The mixture was then extracted from water with $\mathrm{CH}_{2} \mathrm{Cl}_{2}$, the combination of organic phases was dried over magnesium sulfate and the solids were filtered off. The solvent was then removed under reduced pressure, and after chromatography (hexanes:ethyl acetate, increasing gradually the polarity from $3: 1$ to $1: 1$ to $1: 3)$ the title compound 8 was obtained $(19 \mathrm{mg}, 12 \%)$. The structural assignment of $\mathbf{8}$ is based on favorable comparison of proton NMR features with tricyclic 6 and tetracyclic 7, for which crystal structures were obtained to unambiguously establish atom connectivity and stereochemistry. ${ }^{1}$

${ }^{1} \mathrm{H}$ NMR (400 MHz, $\left.\mathrm{CDCl}_{3}\right) \delta 4.18(\mathrm{dd}, J=10.0,6.0 \mathrm{~Hz}, 1 \mathrm{H}), 4.03(\mathrm{t}, J=10.4 \mathrm{~Hz}, 1 \mathrm{H}), 3.96$ $(\mathrm{dd}, J=10.4,6.0 \mathrm{~Hz}, 1 \mathrm{H}), 3.76(\mathrm{~d}, J=6.4 \mathrm{~Hz}, 1 \mathrm{H}), 3.62(\mathrm{dd}, J=11.4,3.0 \mathrm{~Hz}, 1 \mathrm{H}), 3.61(\mathrm{dd}, J=$ 11.4, $5.4 \mathrm{~Hz}, 1 \mathrm{H}), 3.49$ (dd, $J=10.4,4.4 \mathrm{~Hz}, 1 \mathrm{H}), 2.20-1.50(\mathrm{~m}, 16 \mathrm{H}), 1.44(\mathrm{~s}, 3 \mathrm{H}), 1.28(\mathrm{~s}, 3 \mathrm{H})$, $1.24(\mathrm{~s}, 3 \mathrm{H}), 1.22(\mathrm{~s}, 3 \mathrm{H}), 1.20(\mathrm{~s}, 3 \mathrm{H}), 1.08(\mathrm{~s}, 3 \mathrm{H})$.

\footnotetext{
${ }^{1}$ McDonald, F. E.; Bravo, F.; Wang, X.; Wei, X.; Toganoh, M.; Rodríguez, J. R.; Do, B.; Neiwert, W. A.; Hardcastle, K. I. J. Org. Chem. 2002, 67, 2515.
} 


\section{(2R,3R)-1-O-tert-butoxycarbonyl-2,3-epoxy-3,7-dimethyl-octa-6-en-1-ol (11).}

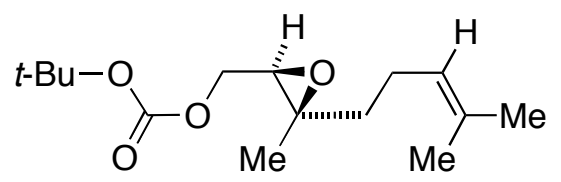

11

Geraniol monoepoxide $^{2}(2.75 \mathrm{~g}, 16.2 \mathrm{mmol})$ was dissolved in anhydrous toluene $(80 \mathrm{~mL})$ and $N$ methylimidazole $(1.70 \mathrm{~mL}, 21.3 \mathrm{mmol})$, and melted $\mathrm{Boc}_{2} \mathrm{O}(14.2 \mathrm{~g}, 64.9 \mathrm{mmol})$ was added dropwise at $0^{\circ} \mathrm{C}$. The reaction was stirred overnight, allowing the temperature to warm to room temperature slowly. The reaction mixture was then diluted with dichloromethane, poured over water, and extracted with more dichloromethane. The combination of organic layers was dried over magnesium sulfate, filtered, and the solvent was removed under reduced pressure. The crude thus obtained was purified by column chromatography (pentane:ethyl ether 20:1, containing $0.5 \%$ of triethylamine) to furnish $11(3.72 \mathrm{~g}, 85 \%)$.

$[\alpha]_{\mathrm{D}}^{25}=+11.3\left(c 1.730, \mathrm{CHCl}_{3}\right)$.

El. anal. calcd. for $\mathrm{C}_{15} \mathrm{H}_{26} \mathrm{O}_{4}$ : C, 66.64; H, 9.69. Found: C, 66.70; H, 9.75.

IR (neat): 2979, 2931, 2860, 1744, 1477, 1455, 1370, 1278, 1255, 1164, 1093, 136, 861, $793 \mathrm{~cm}^{-1}$. ${ }^{1} \mathrm{H}$ NMR (400 MHz, $\mathrm{CDCl}_{3}$ ) $\delta 5.04$ (tquint, $\left.J=7.0,1.4 \mathrm{~Hz}, 1 \mathrm{H}\right), 4.18$ (dd, $J=11.6,4.8 \mathrm{~Hz}, 1 \mathrm{H}$ ), $4.09(\mathrm{dd}, J=11.6,6.4 \mathrm{~Hz}, 1 \mathrm{H}), 2.98(\mathrm{dd}, J=6.4,4.8 \mathrm{~Hz}, 1 \mathrm{H}), 2.06(\mathrm{dd}, J=15.4,7.8 \mathrm{~Hz}, 2 \mathrm{H})$, $1.65(\mathrm{~d}, J=0.8 \mathrm{~Hz}, 3 \mathrm{H}), 1.62(\mathrm{dd}, J=8.4,6.8 \mathrm{~Hz}, 1 \mathrm{H}), 1.57(\mathrm{~s}, 3 \mathrm{H}), 1.47(\mathrm{~s}, 9 \mathrm{H}), 1.48-1.40(\mathrm{~m}$, $2 \mathrm{H}), 1.28(\mathrm{~s}, 3 \mathrm{H})$.

${ }^{13} \mathrm{C}$ NMR $(100.5 \mathrm{MHz}) \delta 153.3,132.1,123.2,82.4,65.5,60.5,59.4,38.1,27.6,25.6,23.5,17.6$, 16.7 .

\footnotetext{
${ }^{2}$ Gao, Y.; Hanson, R. M.; Klunder, J. M.; Ko, S. Y.; Masamune, H.; Sharpless, K. B. J. Am. Chem. Soc. 1987, 109, 5765 .
} 


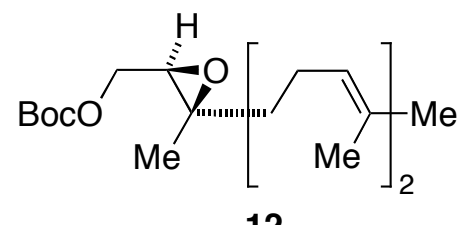

12

The procedure described for the synthesis of $\mathbf{1 1}$ was followed, starting with: farnesol monoepoxide $^{3}(1.88 \mathrm{~g}, 7.87 \mathrm{mmol}), \mathrm{N}$-methylimidazole $(0.75 \mathrm{~mL}, 9.41 \mathrm{mmol}), \mathrm{Boc}_{2} \mathrm{O}(6.84 \mathrm{~g}$, $31.3 \mathrm{mmol})$ in toluene $(200 \mathrm{~mL})$. After the work-up, the reaction was purified by column chromatography (pentane:ethyl ether $20: 1$, containing $0.5 \%$ of $\mathrm{NEt}_{3}$ ), to yield the title compound $12(2.32 \mathrm{~g}, 87 \%)$.

$[\alpha]_{\mathrm{D}}^{25}=+12.7\left(c 1.712, \mathrm{CHCl}_{3}\right)$.

El. anal. calcd. for $\mathrm{C}_{20} \mathrm{H}_{34} \mathrm{O}_{4}$ : C, 70.97; H, 10.12. Found: C, 71.22; H, 10.20.

IR (neat): 2979, 2927, 2858, 1744, 1669, 1455, 1370, 1326, 1278, 1256, 1166, 1093, 1038, 936, $861,793,767 \mathrm{~cm}^{-1}$.

${ }^{1} \mathrm{H}$ NMR (400 MHz, $\mathrm{CDCl}_{3}$ ) $\delta 5.07$ (br m, 2H), 4.20 (dd, $\left.J=7.5,3.0 \mathrm{~Hz}, 1 \mathrm{H}\right), 4.10$ (dd, $J=7.5$, $4.0 \mathrm{~Hz}, 1 \mathrm{H}), 3.01$ (dd, $J=4.0,3.0 \mathrm{~Hz}, 1 \mathrm{H}), 2.10-2.01(\mathrm{~m}, 4 \mathrm{H}), 1.98-1.94(\mathrm{~m}, 2 \mathrm{H}), 1.66(\mathrm{~s}, 3 \mathrm{H})$, $1.58(\mathrm{~s}, 3 \mathrm{H}), 1.48(\mathrm{~s}, 3 \mathrm{H}), 1.30(\mathrm{~s}, 3 \mathrm{H})$.

${ }^{13} \mathrm{C}$ NMR $\left(100.5 \mathrm{MHz}, \mathrm{CDCl}_{3}\right) \delta 153.3,135.8,131.3,124.2,123.0,82.4,65.6,60.5,59.4,39.6$, $38.2,27.7,26.6,25.6,23.4,17.6,16.8,15.9$.

$\left(2 R, 3 R, \Delta^{6,7} E\right)-1-O$-tert-butoxycarbonyl-3,7-dimethyl-2,3-epoxy-octa-6-en-1,8-diol (13).

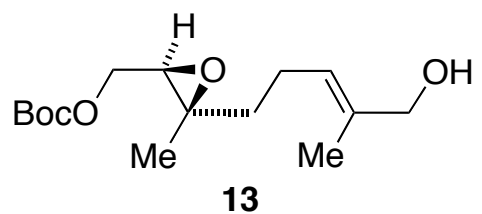

The procedure for the allylic oxidation described above was followed, reacting 11 ( $2.21 \mathrm{~g}, 8.17$ mmol) with $\mathrm{SeO}_{2}(280 \mathrm{mg}, 1.62 \mathrm{mmol}), t-\mathrm{BuOOH}(70 \%$ in water, $2.30 \mathrm{~mL}, 17.9 \mathrm{mmol})$ in

${ }^{3}$ Corey, E. J.; Ha, D.-C. Tetrahedron Lett. 1988, 29, 3171. 
$\mathrm{CH}_{2} \mathrm{Cl}_{2}(80 \mathrm{~mL}$ in total). The reaction was stopped after $36 \mathrm{~h}$ as described before, and the crude was purified by column chromatography (pentane:ethyl ether from 10:1 to $3: 1$, containing $0.5 \%$ $\mathrm{NEt}_{3}$ ), to afford 13 (1.21 g, 52\%), along with $300 \mathrm{mg}$ (14\%) of unreacted 11.

$[\alpha]_{\mathrm{D}}^{25}=+7.7\left(c 0.918, \mathrm{CHCl}_{3}\right)$.

El. anal. calcd. for $\mathrm{C}_{15} \mathrm{H}_{26} \mathrm{O}_{5}$ : C, 62.91; H, 9.15. Found: C, 63.01; H, 9.24.

IR (neat): 3468 (br), 2979, 2932, 2862, 1743, 1634, 1457, 1370, 1327, 1279, 1255, 1161, 1012, $936,858,793,767 \mathrm{~cm}^{-1}$.

${ }^{1} \mathrm{H}$ NMR (300 MHz, $\left.\mathrm{CDCl}_{3}\right) \delta 5.35$ (approx. t, $\left.J=7.2 \mathrm{~Hz}, 1 \mathrm{H}\right), 4.20$ (dd, $\left.J=12.0,4.8 \mathrm{~Hz}, 1 \mathrm{H}\right)$, $4.06(\mathrm{dd}, J=12.0,5.7 \mathrm{~Hz}, 1 \mathrm{H}), 3.96(\mathrm{~s}, 2 \mathrm{H}), 2.98(\mathrm{dd}, J=5.7,4.8 \mathrm{~Hz}, 1 \mathrm{H}), 2.13(\mathrm{dd}, J=14.7$, $7.2 \mathrm{~Hz}, 2 \mathrm{H}), 1.94$ (bs, $l \mathrm{H}), 1.73$ (dd, $J=13.8,6.9 \mathrm{~Hz}, 1 \mathrm{H}), 1.63(\mathrm{~s}, 3 \mathrm{H}), 1.60-1.40(\mathrm{~m}, 2 \mathrm{H}), 1.47$ $(\mathrm{s}, 9 \mathrm{H}), 1.28(\mathrm{~s}, 3 \mathrm{H})$.

${ }^{13} \mathrm{C}$ NMR $(75.4 \mathrm{MHz}) \delta 153.3,135.6,124.2,82.7,68.5,65.5,60.3,59.5,37.7,27.6,23.1,16.6$, 13.6.

(2R,3R, $\left.\Delta^{6,7} E, \Delta^{10,11} E\right)-1-O$-tert-butoxycarbonyl-2,3-epoxy-3,7,11-trimethyl-dodeca-6,10dien-1,12-diol (14).

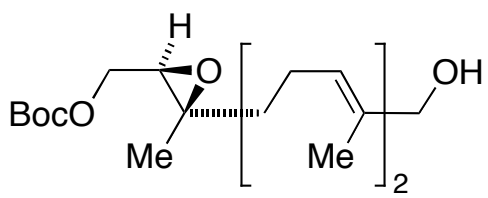

14

The procedure for allylic oxidation described above was followed, reacting 12 (2.63 g, 7.78 mmol) with $\mathrm{SeO}_{2}(90 \mathrm{mg}, 0.81 \mathrm{mmol}), t$ - $\mathrm{BuOOH}(70 \%$ in water, $2.20 \mathrm{~mL}, 17.1 \mathrm{mmol})$ in $\mathrm{CH}_{2} \mathrm{Cl}_{2}$ ( $80 \mathrm{~mL}$ in total). The reaction was stopped after 4 days as described before, and the crude was purified by column chromatography (pentane:ethyl ether, increasing gradually the polarity from $3: 1$ to $2: 1$ to $1: 1$, containing $0.5 \%$ of $\left.\mathrm{NEt}_{3}\right)$, to afford alcohol $14(740 \mathrm{mg}, 27 \%)$ and $518 \mathrm{mg}$ (20\%) of unreacted diene 12.

$[\alpha]_{\mathrm{D}}^{25}=+13.4\left(c 1.780, \mathrm{CHCl}_{3}\right)$.

El. anal. calcd. for $\mathrm{C}_{20} \mathrm{H}_{34} \mathrm{O}_{5}$ : C, 67.77; H, 9.67. Found: C, 67.55; H, 9.72.

IR (neat): 3418 (br), 2978, 2931, 2859, 1743, 1636, 1456, 1370, 1278, 1255, 1161, 1131, 1102, $859,793,767 \mathrm{~cm}^{-1}$. 
${ }^{1} \mathrm{H}$ NMR (400 MHz, $\left.\mathrm{CDCl}_{3}\right) \delta 5.35$ (tq, $\left.J=7.0,1.4 \mathrm{~Hz}, 1 \mathrm{H}\right), 5.08$ (tq, $\left.J=6.9,1.3 \mathrm{~Hz}, 1 \mathrm{H}\right), 4.19$ (dd, $J=11.6,4.8 \mathrm{~Hz}, 1 \mathrm{H}), 4.09$ (dd, $J=11.6,6.2 \mathrm{~Hz}, 1 \mathrm{H}), 3.95$ (s, 1H), 2.99 (dd, $J=6.2,5.0 \mathrm{~Hz}$, $1 \mathrm{H}), 2.16-1.96(\mathrm{~m}, 6 \mathrm{H}), 1.66-1.58(\mathrm{~m}, 1 \mathrm{H}), 1.63(\mathrm{~s}, 3 \mathrm{H}), 1.52-1.40(\mathrm{~m}, 1 \mathrm{H}), 1.58(\mathrm{~s}, 3 \mathrm{H}), 1.47(\mathrm{~s}$, 9H), 1.29 (s, 3H).

${ }^{13} \mathrm{C}$ NMR $(100.5 \mathrm{MHz}) \delta 153.3,135.3,134.8,125.6,123.4,82.5,68.7,65.5,60.6,59.3,39.1$, $38.0,27.6,25.9,23.4,16.7,15.9,13.6$.

$\left(2 R, 3 R, \Delta^{6,7} E\right)-1-O$-tert-butoxycarbonyl-3,7-dimethyl-2,3-epoxy-nona-6,8-dien-1-ol.

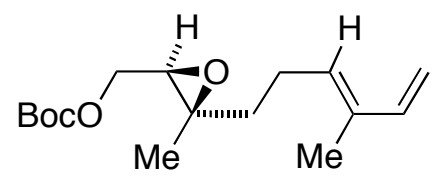

Alcohol 13 (917 mg, $3.20 \mathrm{mmol})$ was dissolved in anhydrous DMSO (13 mL), and IBX (1.26 g, $4.50 \mathrm{mmol}$ ) was added in one portion at room temperature. The reaction was stirred for $30 \mathrm{~min}$., and then diluted with ether. The solid precipitate was filtered off, and the filtrate was then washed with water (three times). The mixture was then dried with magnesium sulfate, then filtered and the solvent removed under vacuum. Traces of water in the crude were azeotropically removed by co-distillation with toluene under reduced pressure, thus affording the crude aldehyde for the next step.

Simultaneously, a suspension of methyltriphenylphosphonium bromide (1.37 g, $3.84 \mathrm{mmol})$ in anhydrous THF (25 mL) was treated with $n$-butyllithium (1.6 M in hexanes, $2.0 \mathrm{~mL}, 3.20 \mathrm{mmol})$ and stirred at room temperature for $1 \mathrm{~h}$. The yellow solution of the ylide thus formed was added to a solution of the aldehyde previously obtained in THF $(7 \mathrm{~mL})$. The reaction was stirred at room temperature for $12 \mathrm{~h}$, then diluted with ether and quenched by adding a few milliliters of $\mathrm{NH}_{4} \mathrm{Cl}$ (aq., sat.) at $0^{\circ} \mathrm{C}$. The two layers were separated, and the aqueous phase was extracted with more ether. The organic fractions were combined and dried over $\mathrm{Na}_{2} \mathrm{SO}_{4}$. The solids were then filtered off, and the volatile solvents removed under reduced pressure. Purification with column chromatography (pentane:ethyl ether 15:1, containing $0.5 \%$ of $\mathrm{NEt}_{3}$ ) afforded pure diene (766 mg, 85\%)

$[\alpha]_{\mathrm{D}}^{25}=+13.2\left(c 0.850, \mathrm{CHCl}_{3}\right)$. 
El. anal. calcd. for $\mathrm{C}_{16} \mathrm{H}_{26} \mathrm{O}_{4}: \mathrm{C}, 68.06 ; \mathrm{H}, 9.28$. Found: C, 68.39; H, 9.36.

IR (neat): 3089, 2981, 2936, 2873, 1743, 1642, 1607, 1478, 1457, 1390, 1370, 1327, 1278, 1255, $1164,1091,990,859,793,767 \mathrm{~cm}^{-1}$.

${ }^{1} \mathrm{H}$ NMR (400 MHz, $\left.\mathrm{CDCl}_{3}\right) \delta 6.33(\mathrm{dd}, J=17.2,10.8 \mathrm{~Hz}, 1 \mathrm{H}$ ), 5.42 (approx. t, $J=7.2 \mathrm{~Hz}, 1 \mathrm{H}$ ), $5.08(\mathrm{~d}, J=17.2 \mathrm{~Hz}, 1 \mathrm{H}), 4.93(\mathrm{~d}, J=10.8 \mathrm{~Hz}, 1 \mathrm{H}), 4.19$ (dd, $J=12.0,4.8 \mathrm{~Hz}, 1 \mathrm{H}), 4.11$ (dd, $J$ $=12.0,6.2 \mathrm{~Hz}, 1 \mathrm{H}), 3.01(\mathrm{dd}, J=6.2,4.8 \mathrm{~Hz}, 1 \mathrm{H}), 2.22(\mathrm{dd}, J=15.4,7.8 \mathrm{~Hz}, 2 \mathrm{H}), 1.72(\mathrm{~s}, 3 \mathrm{H})$, 1.72-1.65 (m, 1H), 1.55 (ddd, $J=14.2,9.0,7.4 \mathrm{~Hz}, 1 \mathrm{H}), 1.48$ (s, 9H), 1.31 (s, 3H).

${ }^{13} \mathrm{C}$ NMR $(100.5 \mathrm{MHz}) \delta 153.2,141.2,134.6,131.3,111.0,82.5,65.4,60.3,59.3,37.7,27.6$, 23.7, 16.8, 11.6.

\section{$(2 R, 3 R, 6 R, 7 R)-1-O$-tert-butoxycarbonyl-2,3;6,7-diepoxy-3,7-dimethyl-nona-8-en-1-ol (15).}

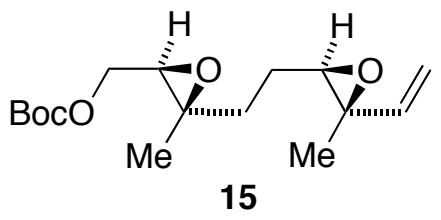

A modification of the procedure described by Shi $e t a l .{ }^{4}$ was followed: The diene 3 (334 mg, $1.18 \mathrm{mmol}$ ) was dissolved in a 1:2 mixture of acetonitrile:dimethoxymethane. Then, under vigorous agitation, a $0.05 \mathrm{M}$ solution of $\mathrm{Na}_{2} \mathrm{~B}_{4} \mathrm{O}_{7}$ in $4 \cdot 10^{-4} \mathrm{M} \mathrm{Na}_{2}$ (EDTA) $(12 \mathrm{~mL}), \mathrm{NBu}_{4} \mathrm{HSO}_{4}$ (17 mg, $0.05 \mathrm{mmol}$ ) and Shi ketone catalyst (335 mg, $1.29 \mathrm{mmol}$ ), were sequentially added. The mixture was cooled to $0^{\circ} \mathrm{C}$, and then Oxone $(730 \mathrm{mg}, 1.19 \mathrm{mmol})$, dissolved in $4 \cdot 10^{-4} \mathrm{M}$ $\mathrm{Na}_{2}$ (EDTA) (7.7 mL), and $\mathrm{K}_{2} \mathrm{CO}_{3}(950 \mathrm{mg}, 6.87 \mathrm{mmol})$, dissolved in water $(7.7 \mathrm{~mL})$, were simultaneously added over the mixture over a $30 \mathrm{~min}$. period of time. Once the addition was completed, the mixture was diluted with water and extracted with dichloromethane. The organic extracts were dried over $\mathrm{MgSO}_{4}$, and the solvent was removed under vacuum. After purification by column chromatography (pentane: ethyl ether, increasing gradually the polarity from 10:1 to 5:1 to 3:1, containing 0.5\% $\mathrm{NEt}_{3}$ ), compound 15 was isolated (193 mg, 55\%), along with $81 \mathrm{mg}$ of unreacted diene (24\%).

\footnotetext{
4 (a) Wang, Z.; Tu, Y.; Frohn, M.; Zhang, J.; Shi, Y. J. Am. Chem. Soc. 1997, 119, 11224. (b) Cao, G.-A.; Wang, Z.X.; Tu, Y.; Shi, Y. Tetrahedron Lett. 1998, 39, 4425. (c) Zhu, Y.; Tu, Y.; Tu, H.; Shi, Y. Tetrahedron Lett. 1998, 39 , 7819. (d) Shi, Y.; Wang, Z.-X. J. Org. Chem. 1998, 63, 3099.
} 
$[\alpha]_{\mathrm{D}}^{25}=+16.2\left(c 1.800, \mathrm{CHCl}_{3}\right)$.

El. anal. calcd. for $\mathrm{C}_{16} \mathrm{H}_{26} \mathrm{O}_{5}$ : C, 64.41; H, 8.78. Found: C, 64.30; H, 8.78.

IR (neat): 3091, 2979, 2936, 2875, 1745, 1641, 1457, 1370, 1328, 1280, 1257, 1165, 1089, 990, $924,861,793,768 \mathrm{~cm}^{-1}$.

${ }^{1} \mathrm{H}$ NMR (400 MHz, $\left.\mathrm{CDCl}_{3}\right) \delta 5.56(\mathrm{dd}, J=17.4,10.6 \mathrm{~Hz}, 1 \mathrm{H}), 5.52(\mathrm{dd}, J=17.4,1.4 \mathrm{~Hz}, 1 \mathrm{H})$, $5.08(\mathrm{dd}, J=10.6,1.0 \mathrm{~Hz}, 1 \mathrm{H}), 4.12(\mathrm{dd}, J=12.0,5.2 \mathrm{~Hz}, 1 \mathrm{H}), 4.05(\mathrm{dd}, J=12.0,6.0 \mathrm{~Hz}, 1 \mathrm{H})$, $2.95(\mathrm{dd}, J=6.0,5.2 \mathrm{~Hz}, 1 \mathrm{H}), 2.72-2.66(\mathrm{~m}, 1 \mathrm{H}), 1.76-1.70(\mathrm{~m}, 1 \mathrm{H}), 1.65-1.55(\mathrm{~m}, 3 \mathrm{H}), 1.40$ (s, 9H), $1.30(\mathrm{~s}, 3 \mathrm{H}), 1.25(\mathrm{~s}, 3 \mathrm{H})$.

${ }^{13} \mathrm{C}$ NMR $(100.5 \mathrm{MHz}) \delta 153.0,140.4,115.7,82.3,65.2,65.1,64.4,64.2,59.8,59.3,58.9,34.4$, $27.5,24.0,16.7,14.7$.

$\left(2 R, 3 R, 10 R, 11 R, \Delta^{6,7} E\right)-1-O$-tert-butoxycarbonyl-2,3;10,11-diepoxy-3,7,11-trimethyl-dodeca6-en-1,12-diol.

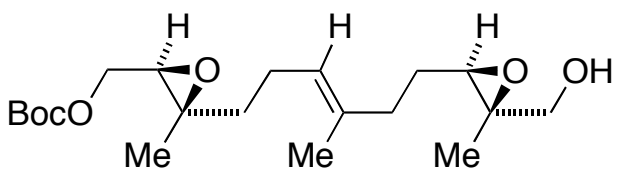

The Sharpless epoxidation was carried out as described above, with the following amounts of reagents: $4 \AA$ M.S. (600 mg), D-(-)-DIPT (60 $\mu \mathrm{L}, 0.29 \mathrm{mmol}), \operatorname{Ti}(\mathrm{O} i \text {-Pr })_{4}(60 \mu \mathrm{L}, 0.20 \mathrm{mmol}), t$ $\mathrm{BuOOH}(5.0-6.0 \mathrm{M}$ in decane, $550 \mu \mathrm{L}, 3.02 \mathrm{mmol})$, and the allylic alcohol 14 (705 $\mathrm{mg}, 1.99$ $\mathrm{mmol})$ in dichloromethane $\left(20 \mathrm{~mL}\right.$ in total). The reaction was kept at $-18^{\circ} \mathrm{C}$ overnight, and was quenched by addition of acetone containing $2 \%$ water $(10 \mathrm{~mL})$, warmed at room temperature and stirred for 3h. After filtering through Celite, the solvent was dried over $\mathrm{MgSO}_{4}$, the solids were filtered off, and the solvent evaporated under vacuum. The crude mixture was purified by column chromatography (pentane: ethyl ether 1:1), to provide the title compound (635 $\mathrm{mg}, 86 \%$ ). $[\alpha]_{\mathrm{D}}^{25}=+20.9\left(c 1.655, \mathrm{CHCl}_{3}\right)$.

El. anal. calcd. for $\mathrm{C}_{20} \mathrm{H}_{34} \mathrm{O}_{6}$ : C, 64.84; H, 9.25. Found C, 64.31; H, 9.31.

IR (neat): 3478 (br), 2973, 2929, 2873, 1743, 1644, 1457, 1370, 1279, 1256, 1164, 1094, 1038, $936,859,793,766 \mathrm{~cm}^{-1}$. 
${ }^{1} \mathrm{H}$ NMR (400 MHz, $\mathrm{CDCl}_{3}$ ) $\delta 5.13$ (tq, $\left.J=7.0,1.3 \mathrm{~Hz}, 1 \mathrm{H}\right), 4.20$ (dd, $\left.J=11.8,4.8 \mathrm{~Hz}, 1 \mathrm{H}\right)$, $4.09(\mathrm{dd}, J=11.8,6.4 \mathrm{~Hz}, 1 \mathrm{H}), 3.64$ (d, $J=12.0 \mathrm{~Hz}, 1 \mathrm{H}), 3.00(\mathrm{dd}, J=6.2,4.6 \mathrm{~Hz}, 1 \mathrm{H}), 2.99$ (t, $J=6.4 \mathrm{~Hz}, 1 \mathrm{H}), 1.93(\mathrm{bs}, 1 \mathrm{H}), 1.72-1.62(\mathrm{~m}, 3 \mathrm{H}), 1.61(\mathrm{~s}, 3 \mathrm{H}), 1.52(\mathrm{dd}, J=9.4,6.6 \mathrm{~Hz}, 1 \mathrm{H})$, $1.48(\mathrm{~s}, 9 \mathrm{H}), 1.29(\mathrm{~s}, 3 \mathrm{H}), 1.26(\mathrm{~s}, 3 \mathrm{H})$.

${ }^{13} \mathrm{C}$ NMR $(100.5 \mathrm{MHz}) \delta 153.3,134.7,123.9,82.5,65.5,65.4,60.9,60.5,59.7,59.3,38.0,36.2$, $27.7,26.6,23.4,16.8,15.9,14.2$.

\section{$(2 R, 3 R, 6 R, 7 R, 10 R, 11 R)-1-O$-tert-butoxycarbonyl-2,3;6,7;10,11-triepoxy-3,7,11-trimethyl-} dodeca-1,12-diol.

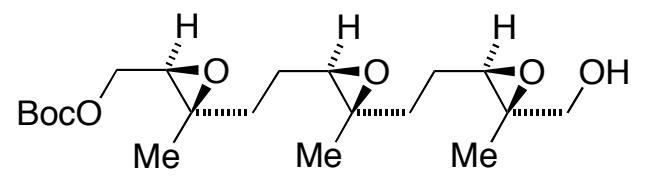

The Shi epoxidation was conducted as described for the synthesis of $\mathbf{4}$, using the following amounts of reagents: the alkene previously obtained $(362 \mathrm{mg}, 1.00 \mathrm{mmol})$, a 1:2 mixture of acetonitrile: dimethoxymethane $(15 \mathrm{~mL})$, a $0.05 \mathrm{M}$ solution of $\mathrm{Na}_{2} \mathrm{~B}_{4} \mathrm{O}_{7}$ in $4 \cdot 10^{-4} \mathrm{M} \mathrm{Na}_{2}(\mathrm{EDTA})$ (10 mL), $\mathrm{NBu}_{4} \mathrm{HSO}_{4}(14 \mathrm{mg}, 0.04 \mathrm{mmol})$, Shi catalyst (76 mg, $\left.0.29 \mathrm{mmol}\right)$, Oxone (830 mg, $1.35 \mathrm{mmol}$ ), dissolved in $4 \cdot 10^{-4} \mathrm{M} \mathrm{Na}_{2}$ (EDTA) $(6.4 \mathrm{~mL})$, and $\mathrm{K}_{2} \mathrm{CO}_{3}$ (785 $\mathrm{mg}, 5.68 \mathrm{mmol}$ ), dissolved in water $(6.4 \mathrm{~mL})$. After the work-up and purification by column chromatography (pentane:ethyl ether 1:3, containing 0.5\% $\mathrm{NEt}_{3}$ ), the title compound was isolated (340 $\left.\mathrm{mg}, 90 \%\right)$. $[\alpha]_{\mathrm{D}}^{25}=+75.2\left(c 1.128, \mathrm{CHCl}_{3}\right)$.

El. anal. calcd. for $\mathrm{C}_{20} \mathrm{H}_{34} \mathrm{O}_{7}$ : C, 62.16; H, 8.87. Found C, 62.24; H, 8.73.

IR (neat): 3437 (br), 2974, 2933, 2873, 1742, 1460, 1386, 1370, 1279, 1256, 1163, 1093, 1038, $961,936,859,793,767 \mathrm{~cm}^{-1}$.

${ }^{1} \mathrm{H}$ NMR $\left(400 \mathrm{MHz}, \mathrm{CDCl}_{3}\right) \delta 4.19(\mathrm{dd}, J=12.0,4.8 \mathrm{~Hz}, 1 \mathrm{H}), 4.12(\mathrm{dd}, J=12.0,6.0 \mathrm{~Hz}, 1 \mathrm{H})$, $3.61(\mathrm{dd}, J=12.0,4.4 \mathrm{~Hz}, 1 \mathrm{H}), 3.56(\mathrm{dd}, J=12.0,8.2 \mathrm{~Hz}, 1 \mathrm{H}), 3.05-2.96(\mathrm{~m}, 2 \mathrm{H}), 2.76-2.69$ (m, 1H), 1.98 (dd, $J=8.2,4.6 \mathrm{~Hz}, 1 \mathrm{H}), 1.80$ (bs, 1H), 1.76-1.56 (m, 7H), 1.47 (s, 9H), 1.31 (s, 3H), 1.27 (s, 3H), 1.26 (s, 3H).

${ }^{13} \mathrm{C}$ NMR $(100.5 \mathrm{MHz}) \delta 153.2,82.6,65.6,65.3,62.5,60.9,60.4,60.0,59.6,59.0,35.0,34.6$, $27.7,24.0,23.9,16.9,16.4,14.1$. 

trideca-12-en-1-ol (16).

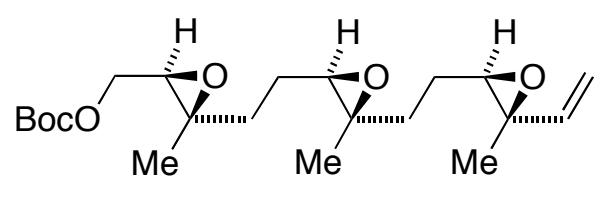

16

The alcohol obtained in the previous step $(212 \mathrm{mg}, 0.55 \mathrm{mmol})$ was dissolved in anhydrous dichloromethane $(5.5 \mathrm{~mL})$ and triethylamine $(460 \mu \mathrm{L})$, and a solution of $\mathrm{SO}_{3} \cdot \mathrm{py}(260 \mathrm{mg}, 1.63$ mmol) in DMSO $(900 \mu \mathrm{L})$ was added dropwise at $0^{\circ} \mathrm{C}$. The reaction was stirred for $3 \mathrm{~h}$, and then the solvent was evaporated. The crude was then filtered through a short column of silica gel (approx. $5 \mathrm{~cm}$, pentane:ethyl ether 1:1, containing $0.5 \% \mathrm{NEt}_{3}$, as solvent system). The solvent was evaporated under reduced pressure, and traces of water were azeotropically removed by codistillation with toluene under reduced pressure, thus affording the crude aldehyde for the next step.

Simultaneously, a suspension of methyltriphenylphosphonium bromide $(235 \mathrm{mg}, 0.66 \mathrm{mmol})$ in anhydrous THF ( $4.1 \mathrm{~mL})$ was treated with potassium tert-butoxide (1.0 M in THF, $0.58 \mathrm{~mL}, 0.58$ $\mathrm{mmol}$ ) and stirred at room temperature for $1 \mathrm{~h}$. The yellow solution of the ylide thus formed was added to a solution of the aldehyde previously obtained in THF (1.4 mL). The reaction was stirred at room temperature for $12 \mathrm{~h}$, then diluted with ether and quenched by adding a few milliliters of $\mathrm{NH}_{4} \mathrm{Cl}$ (aq., sat.) at $0^{\circ} \mathrm{C}$. The two layers were separated, and the aqueous phase was extracted with more ether. The organic fractions were combined and dried over $\mathrm{MgSO}_{4}$. The solids were then filtered off, and the volatile solvents removed under reduced pressure. Purification with column chromatography (pentane:ethyl ether 3:1, containing $0.5 \%$ of $^{-N_{3}}$ ) afforded pure 16 (142 mg, 68\%).

$[\alpha]_{\mathrm{D}}^{25}=+22.2\left(c 0.810, \mathrm{CHCl}_{3}\right)$.

El. anal. calcd. for $\mathrm{C}_{20} \mathrm{H}_{34} \mathrm{O}_{7}$ : C, 65.94; H, 8.96. Found: C, 65.78; H, 8.99.

IR (neat): 3091, 2972, 2934, 2873, 1743, 1640, 1459, 1386, 1370, 1279, 1256, 1164, 1092, 991, $923,861,793,767 \mathrm{~cm}^{-1}$.

${ }^{1} \mathrm{H}$ NMR (400 MHz, $\left.\mathrm{CDCl}_{3}\right) \delta 5.63(\mathrm{dd}, J=17.6,10.8 \mathrm{~Hz}, 1 \mathrm{H}), 5.30(\mathrm{dd}, J=17.6,1.0 \mathrm{~Hz}, 1 \mathrm{H})$, $5.17(\mathrm{dd}, J=10.8,1.0 \mathrm{~Hz}, 1 \mathrm{H}), 4.20(\mathrm{dd}, J=12.0,5.0 \mathrm{~Hz}, 1 \mathrm{H}), 4.13(\mathrm{dd}, J=12.0,6.0 \mathrm{~Hz}, 1 \mathrm{H})$, 
$3.02(\mathrm{dd}, J=6.2,5.0 \mathrm{~Hz}, 1 \mathrm{H}), 2.77(\mathrm{t}, J=6.0 \mathrm{~Hz}, 1 \mathrm{H}), 2.73(\mathrm{t}, J=6.0 \mathrm{~Hz}, 1 \mathrm{H}), 1.82-1.56(\mathrm{~m}$, $8 \mathrm{H}), 1.48(\mathrm{~s}, 9 \mathrm{H}), 1.38(\mathrm{~s}, 3 \mathrm{H}), 1.32(\mathrm{~s}, 3 \mathrm{H}), 1.26(\mathrm{~s}, 3 \mathrm{H})$.

${ }^{13} \mathrm{C}$ NMR (100.5 MHz) $\delta$ 153.2, 140.7, 116.0, 82.6, 65.3, 64.6, 62.5, 60.3, 60.1, 59.6, 59.0, 35.0, $34.6,27.7,24.3,24.1,16.9,16.6,14.9$.

\section{Bicyclic 17.}

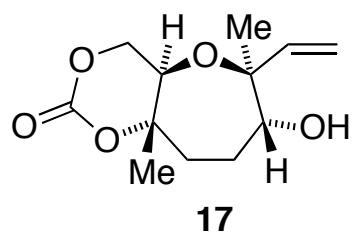

The diepoxide 15 (44 mg, $0.15 \mathrm{mmol})$ was dissolved in anhydrous dichloromethane $(2.9 \mathrm{~mL})$, and ytterbium (III) triflate $(275 \mathrm{mg}, 0.44 \mathrm{mmol}$ ) was added in one portion at room temperature. The reaction was stirred for 1 hour, and then $10 \mathrm{~mL}$ of $\mathrm{NaHCO}_{3}$ were added. The mixture was poured into water and extracted with $\mathrm{CH}_{2} \mathrm{Cl}_{2}$. The organic phases were combined, dried over $\mathrm{MgSO}_{4}$, and the solvent was removed under reduced pressure. Compound 17 (26 mg, 73\%) was isolated after purification by column chromatography (hexanes:ethyl acetate, increasing gradually the polarity from $3: 1$ to $2: 1$ to $1: 1$ to $1: 3$ ).

m.p.: $142-144^{\circ} \mathrm{C}$.

$[\alpha]_{\mathrm{D}}^{25}=+7.1\left(c 0.870, \mathrm{CHCl}_{3}\right)$.

El. anal. calcd. for $\mathrm{C}_{12} \mathrm{H}_{18} \mathrm{O}_{5}: \mathrm{C}, 59.83 ; \mathrm{H}, 7.49$. Found: C, 59.79; H, 7.62.

IR (neat): 3460 (br), 3094, 3084, 1731, 1644, 1540, 1486, 1462, 1438, 1409, 1386, 1353, 1257 , $1225,1123,1108,1068,1046,1018,997,978,928,900,820,770,740,715 \mathrm{~cm}^{-1}$.

${ }^{1} \mathrm{H}$ NMR (600 MHz, $\left.\mathrm{CDCl}_{3}\right) \delta 5.67(\mathrm{dd}, J=17.1,11.1 \mathrm{~Hz}, 1 \mathrm{H}), 5.25(\mathrm{dd}, J=17.1,0.9 \mathrm{~Hz}, 1 \mathrm{H})$, 5.08 (dd, $J=11.1,1.5 \mathrm{~Hz}, 1 \mathrm{H}), 4.49$ (dd, $J=10.8,6.6 \mathrm{~Hz}, 1 \mathrm{H}), 4.19$ (dd, $J=10.8,6.0 \mathrm{~Hz}, 1 \mathrm{H}$ ), $4.06(\mathrm{t}, J=10.8 \mathrm{~Hz}, 1 \mathrm{H}), 3.93(\mathrm{~d}, J=7.2 \mathrm{~Hz}, 1 \mathrm{H}), 2.36$ (bs $1 \mathrm{H}), 3.93(\mathrm{td}, J=14.0,2.7 \mathrm{~Hz}, 1 \mathrm{H})$, $1.86-1.70(\mathrm{~m}, 1 \mathrm{H}), 1.74-1.67(\mathrm{~m}, 2 \mathrm{H}), 1.46(\mathrm{~s}, 3 \mathrm{H}), 1.31(\mathrm{~s}, 3 \mathrm{H})$.

${ }^{13} \mathrm{C}$ NMR $(150.8 \mathrm{MHz}) \delta 148.4,141.8,113.8,84.2,81.7,73.7,66.8,64.2,32.9,23.6,21.1,19.1$. 
The synthesis of bicyclic product $\mathbf{1 7}$ was initially discovered in the enantiomeric series. A crystal structure was obtained for ent-17, whose data are presented below:

Table 1. Crystal data and structure refinement for ent-17.

Identification code

Empirical formula

Formula weight

Temperature

Wavelength

Crystal system

Space group

Unit cell dimensions

Volume

Z

Density (calculated)

Absorption coefficient

$\mathrm{F}(000)$

Crystal size

Theta range for data collection

Index ranges

Reflections collected

Independent reflections

Completeness to theta $=66.29^{\circ}$

Absorption correction

Max. and min. transmission

Refinement method

Data / restraints / parameters

Goodness-of-fit on $\mathrm{F}^{2}$

Final R indices [I $>2 \operatorname{sigma}(\mathrm{I})]$

$\mathrm{R}$ indices (all data)

Absolute structure parameter

Largest diff. peak and hole fb1-288as

C12 H18 O5

242.26

100(2) K

$1.54178 \AA$

Monoclinic

P2(1)

$\mathrm{a}=8.6269(3) \AA$ $\alpha=90^{\circ}$.

$\mathrm{b}=6.7220(2) \AA$ $\beta=92.173(2)^{\circ}$.

$\mathrm{c}=10.4529(3) \AA$ $\gamma=90^{\circ}$.
2

$1.328 \mathrm{Mg} / \mathrm{m}^{3}$

$0.862 \mathrm{~mm}^{-1}$

260

$0.48 \times 0.10 \times 0.03 \mathrm{~mm}^{3}$

4.23 to $66.29^{\circ}$.

$-8<=\mathrm{h}<=9,-7<=\mathrm{k}<=7,-12<=\mathrm{l}<=10$

2801

$1659[\mathrm{R}(\mathrm{int})=0.0173]$

$89.8 \%$

Semi-empirical from equivalents

1.000 and 0.858342

Full-matrix least-squares on $\mathrm{F}^{2}$

$1659 / 1 / 157$

1.010

$\mathrm{R} 1=0.0375, \mathrm{wR} 2=0.1341$

$\mathrm{R} 1=0.0401, \mathrm{w} 22=0.1563$

$-0.1(3)$

0.269 and -0.314 e. $\AA^{-3}$ 
Table 2. Atomic coordinates $\left(\times 10^{4}\right)$ and equivalent isotropic displacement parameters $\left(\AA^{2} \times 10^{3}\right)$ for ent-17. $U(\mathrm{eq})$ is defined as one third of the trace of the orthogonalized $\mathrm{U}^{\mathrm{ij}}$ tensor.

\begin{tabular}{lrrrr}
\hline & $\mathrm{x}$ & $\mathrm{y}$ & $\mathrm{z}$ & $\mathrm{U}(\mathrm{eq})$ \\
\hline $\mathrm{C}(1)$ & $10670(3)$ & $1194(4)$ & $2517(3)$ & $19(1)$ \\
$\mathrm{C}(2)$ & $8188(3)$ & $1833(5)$ & $1367(3)$ & $21(1)$ \\
$\mathrm{C}(3)$ & $7930(3)$ & $3354(4)$ & $2401(2)$ & $17(1)$ \\
$\mathrm{C}(4)$ & $5663(3)$ & $5432(5)$ & $2726(3)$ & $20(1)$ \\
$\mathrm{C}(5)$ & $6380(3)$ & $6194(5)$ & $4003(2)$ & $21(1)$ \\
$\mathrm{C}(6)$ & $7957(3)$ & $7217(4)$ & $3840(3)$ & $20(1)$ \\
$\mathrm{C}(7)$ & $9351(3)$ & $5804(4)$ & $3877(2)$ & $19(1)$ \\
$\mathrm{C}(8)$ & $9458(3)$ & $4457(4)$ & $2715(2)$ & $17(1)$ \\
$\mathrm{C}(9)$ & $10101(3)$ & $5533(4)$ & $1572(3)$ & $21(1)$ \\
$\mathrm{C}(10)$ & $4459(4)$ & $3799(5)$ & $2927(3)$ & $27(1)$ \\
$\mathrm{C}(11)$ & $4890(3)$ & $7143(5)$ & $2022(3)$ & $25(1)$ \\
$\mathrm{C}(12)$ & $5270(4)$ & $7846(5)$ & $902(3)$ & $27(1)$ \\
$\mathrm{O}(1)$ & $10578(2)$ & $2909(3)$ & $3133(2)$ & $20(1)$ \\
$\mathrm{O}(2)$ & $9609(2)$ & $692(3)$ & $1617(2)$ & $23(1)$ \\
$\mathrm{O}(3)$ & $6820(2)$ & $4679(3)$ & $1872(2)$ & $18(1)$ \\
$\mathrm{O}(4)$ & $5474(2)$ & $4592(3)$ & $4904(2)$ & $23(1)$ \\
$\mathrm{O}(5)$ & & & \\
\hline & & & & \\
\hline
\end{tabular}


Table 3. Bond lengths $[\AA]$ and angles $\left[{ }^{\circ}\right]$ for ent-17.

\begin{tabular}{|c|c|c|c|}
\hline $\mathrm{C}(1)-\mathrm{O}(4)$ & $1.211(3)$ & $\mathrm{O}(4)-\mathrm{C}(1)-\mathrm{O}(1)$ & $120.0(2)$ \\
\hline $\mathrm{C}(1)-\mathrm{O}(1)$ & $1.324(4)$ & $\mathrm{O}(4)-\mathrm{C}(1)-\mathrm{O}(2)$ & $119.2(3)$ \\
\hline $\mathrm{C}(1)-\mathrm{O}(2)$ & $1.331(3)$ & $\mathrm{O}(1)-\mathrm{C}(1)-\mathrm{O}(2)$ & $120.8(2)$ \\
\hline $\mathrm{C}(2)-\mathrm{O}(2)$ & $1.461(3)$ & $\mathrm{O}(2)-\mathrm{C}(2)-\mathrm{C}(3)$ & $111.9(2)$ \\
\hline $\mathrm{C}(2)-\mathrm{C}(3)$ & $1.510(4)$ & $\mathrm{O}(2)-\mathrm{C}(2)-\mathrm{H}(2 \mathrm{~A})$ & 109.2 \\
\hline $\mathrm{C}(2)-\mathrm{H}(2 \mathrm{~A})$ & 0.9900 & $\mathrm{C}(3)-\mathrm{C}(2)-\mathrm{H}(2 \mathrm{~A})$ & 109.2 \\
\hline $\mathrm{C}(2)-\mathrm{H}(2 \mathrm{~B})$ & 0.9900 & $\mathrm{O}(2)-\mathrm{C}(2)-\mathrm{H}(2 \mathrm{~B})$ & 109.2 \\
\hline $\mathrm{C}(3)-\mathrm{O}(3)$ & $1.406(3)$ & $\mathrm{C}(3)-\mathrm{C}(2)-\mathrm{H}(2 \mathrm{~B})$ & 109.2 \\
\hline$C(3)-C(8)$ & $1.537(4)$ & $\mathrm{H}(2 \mathrm{~A})-\mathrm{C}(2)-\mathrm{H}(2 \mathrm{~B})$ & 107.9 \\
\hline $\mathrm{C}(3)-\mathrm{H}(3)$ & 1.0000 & $\mathrm{O}(3)-\mathrm{C}(3)-\mathrm{C}(2)$ & $105.3(2)$ \\
\hline $\mathrm{C}(4)-\mathrm{O}(3)$ & $1.455(3)$ & $\mathrm{O}(3)-\mathrm{C}(3)-\mathrm{C}(8)$ & $110.1(2)$ \\
\hline$C(4)-C(11)$ & $1.508(4)$ & $\mathrm{C}(2)-\mathrm{C}(3)-\mathrm{C}(8)$ & $109.3(2)$ \\
\hline$C(4)-C(10)$ & $1.531(4)$ & $\mathrm{O}(3)-\mathrm{C}(3)-\mathrm{H}(3)$ & 110.7 \\
\hline $\mathrm{C}(4)-\mathrm{C}(5)$ & $1.538(3)$ & $\mathrm{C}(2)-\mathrm{C}(3)-\mathrm{H}(3)$ & 110.7 \\
\hline $\mathrm{C}(5)-\mathrm{O}(5)$ & $1.431(3)$ & $\mathrm{C}(8)-\mathrm{C}(3)-\mathrm{H}(3)$ & 110.7 \\
\hline $\mathrm{C}(5)-\mathrm{C}(6)$ & $1.540(4)$ & $\mathrm{O}(3)-\mathrm{C}(4)-\mathrm{C}(11)$ & $105.4(2)$ \\
\hline $\mathrm{C}(5)-\mathrm{H}(5)$ & 1.0000 & $\mathrm{O}(3)-\mathrm{C}(4)-\mathrm{C}(10)$ & $108.6(2)$ \\
\hline$C(6)-C(7)$ & $1.532(4)$ & $C(11)-C(4)-C(10)$ & $108.9(2)$ \\
\hline $\mathrm{C}(6)-\mathrm{H}(6 \mathrm{~A})$ & 0.9900 & $\mathrm{O}(3)-\mathrm{C}(4)-\mathrm{C}(5)$ & $112.7(2)$ \\
\hline $\mathrm{C}(6)-\mathrm{H}(6 \mathrm{~B})$ & 0.9900 & $\mathrm{C}(11)-\mathrm{C}(4)-\mathrm{C}(5)$ & $109.0(2)$ \\
\hline $\mathrm{C}(7)-\mathrm{C}(8)$ & $1.521(4)$ & $C(10)-C(4)-C(5)$ & $111.9(2)$ \\
\hline $\mathrm{C}(7)-\mathrm{H}(7 \mathrm{~A})$ & 0.9900 & $\mathrm{O}(5)-\mathrm{C}(5)-\mathrm{C}(4)$ & $109.4(2)$ \\
\hline $\mathrm{C}(7)-\mathrm{H}(7 \mathrm{~B})$ & 0.9900 & $\mathrm{O}(5)-\mathrm{C}(5)-\mathrm{C}(6)$ & $112.4(2)$ \\
\hline $\mathrm{C}(8)-\mathrm{O}(1)$ & $1.475(3)$ & $C(4)-C(5)-C(6)$ & $112.4(2)$ \\
\hline $\mathrm{C}(8)-\mathrm{C}(9)$ & $1.519(4)$ & $\mathrm{O}(5)-\mathrm{C}(5)-\mathrm{H}(5)$ & 107.5 \\
\hline $\mathrm{C}(9)-\mathrm{H}(9 \mathrm{~A})$ & 0.9800 & $\mathrm{C}(4)-\mathrm{C}(5)-\mathrm{H}(5)$ & 107.5 \\
\hline $\mathrm{C}(9)-\mathrm{H}(9 \mathrm{~B})$ & 0.9800 & $\mathrm{C}(6)-\mathrm{C}(5)-\mathrm{H}(5)$ & 107.5 \\
\hline $\mathrm{C}(9)-\mathrm{H}(9 \mathrm{C})$ & 0.9800 & $C(7)-C(6)-C(5)$ & $114.6(2)$ \\
\hline $\mathrm{C}(10)-\mathrm{H}(10 \mathrm{~A})$ & 0.9800 & $\mathrm{C}(7)-\mathrm{C}(6)-\mathrm{H}(6 \mathrm{~A})$ & 108.6 \\
\hline $\mathrm{C}(10)-\mathrm{H}(10 \mathrm{~B})$ & 0.9800 & $\mathrm{C}(5)-\mathrm{C}(6)-\mathrm{H}(6 \mathrm{~A})$ & 108.6 \\
\hline $\mathrm{C}(10)-\mathrm{H}(10 \mathrm{C})$ & 0.9800 & $\mathrm{C}(7)-\mathrm{C}(6)-\mathrm{H}(6 \mathrm{~B})$ & 108.6 \\
\hline$C(11)-C(12)$ & $1.316(4)$ & $\mathrm{C}(5)-\mathrm{C}(6)-\mathrm{H}(6 \mathrm{~B})$ & 108.6 \\
\hline $\mathrm{C}(11)-\mathrm{H}(11)$ & 0.9500 & $\mathrm{H}(6 \mathrm{~A})-\mathrm{C}(6)-\mathrm{H}(6 \mathrm{~B})$ & 107.6 \\
\hline $\mathrm{C}(12)-\mathrm{H}(12 \mathrm{~A})$ & 0.9500 & $C(8)-C(7)-C(6)$ & $114.9(2)$ \\
\hline $\mathrm{C}(12)-\mathrm{H}(12 \mathrm{~B})$ & 0.9500 & $\mathrm{C}(8)-\mathrm{C}(7)-\mathrm{H}(7 \mathrm{~A})$ & 108.5 \\
\hline $\mathrm{O}(5)-\mathrm{H}(5 \mathrm{~A})$ & 0.8400 & $\mathrm{C}(6)-\mathrm{C}(7)-\mathrm{H}(7 \mathrm{~A})$ & 108.5 \\
\hline
\end{tabular}




$\begin{array}{ll}\mathrm{C}(8)-\mathrm{C}(7)-\mathrm{H}(7 \mathrm{~B}) & 108.5 \\ \mathrm{C}(6)-\mathrm{C}(7)-\mathrm{H}(7 \mathrm{~B}) & 108.5 \\ \mathrm{H}(7 \mathrm{~A})-\mathrm{C}(7)-\mathrm{H}(7 \mathrm{~B}) & 107.5 \\ \mathrm{O}(1)-\mathrm{C}(8)-\mathrm{C}(9) & 108.3(2) \\ \mathrm{O}(1)-\mathrm{C}(8)-\mathrm{C}(7) & 104.03(19) \\ \mathrm{C}(9)-\mathrm{C}(8)-\mathrm{C}(7) & 112.3(2) \\ \mathrm{O}(1)-\mathrm{C}(8)-\mathrm{C}(3) & 105.6(2) \\ \mathrm{C}(9)-\mathrm{C}(8)-\mathrm{C}(3) & 113.4(2) \\ \mathrm{C}(7)-\mathrm{C}(8)-\mathrm{C}(3) & 112.3(2) \\ \mathrm{C}(8)-\mathrm{C}(9)-\mathrm{H}(9 \mathrm{~A}) & 109.5 \\ \mathrm{C}(8)-\mathrm{C}(9)-\mathrm{H}(9 \mathrm{~B}) & 109.5 \\ \mathrm{H}(9 \mathrm{~A})-\mathrm{C}(9)-\mathrm{H}(9 \mathrm{~B}) & 109.5 \\ \mathrm{C}(8)-\mathrm{C}(9)-\mathrm{H}(9 \mathrm{C}) & 109.5 \\ \mathrm{H}(9 \mathrm{~A})-\mathrm{C}(9)-\mathrm{H}(9 \mathrm{C}) & 109.5 \\ \mathrm{H}(9 \mathrm{~B})-\mathrm{C}(9)-\mathrm{H}(9 \mathrm{C}) & 109.5 \\ \mathrm{C}(4)-\mathrm{C}(10)-\mathrm{H}(10 \mathrm{~A}) & 109.5 \\ \mathrm{C}(4)-\mathrm{C}(10)-\mathrm{H}(10 \mathrm{~B}) & 109.5 \\ \mathrm{H}(10 \mathrm{~A})-\mathrm{C}(10)-\mathrm{H}(10 \mathrm{~B}) & 109.5 \\ \mathrm{C}(4)-\mathrm{C}(10)-\mathrm{H}(10 \mathrm{C}) & 109.5 \\ \mathrm{H}(10 \mathrm{~A})-\mathrm{C}(10)-\mathrm{H}(10 \mathrm{C}) & 109.5 \\ \mathrm{H}(10 \mathrm{~B})-\mathrm{C}(10)-\mathrm{H}(10 \mathrm{C}) & 109.5 \\ \mathrm{C}(12)-\mathrm{C}(11)-\mathrm{C}(4) & 126.0(3) \\ \mathrm{C}(12)-\mathrm{C}(11)-\mathrm{H}(11) & 117.0 \\ \mathrm{C}(4)-\mathrm{C}(11)-\mathrm{H}(11) & 117.0 \\ \mathrm{C}(11)-\mathrm{C}(12)-\mathrm{H}(12 \mathrm{~A}) & 120.0 \\ \mathrm{C}(11)-\mathrm{C}(12)-\mathrm{H}(12 \mathrm{~B}) & 120.0 \\ \mathrm{H}(12 \mathrm{~A})-\mathrm{C}(12)-\mathrm{H}(12 \mathrm{~B}) & 120.0 \\ \mathrm{C}(1)-\mathrm{O}(1)-\mathrm{C}(8) & 121.4(2) \\ \mathrm{C}(1)-\mathrm{O}(2)-\mathrm{C}(2) & 122.9(2) \\ \mathrm{C}(3)-\mathrm{O}(3)-\mathrm{C}(4) & 116.83(19) \\ \mathrm{C}(5)-\mathrm{O}(5)-\mathrm{H}(5 \mathrm{~A}) & 109.5 \\ & \end{array}$

Symmetry transformations used to generate equivalent atoms: 
Table 4. Anisotropic displacement parameters $\left(\AA^{2} \times 10^{3}\right)$ for ent-17. The anisotropic displacement factor exponent takes the form: $-2 \pi^{2}\left[h^{2} a^{* 2} U^{11}+\ldots+2 h k a^{*} b^{*} U^{12}\right]$

\begin{tabular}{lllllll}
\hline & $\mathrm{U}^{11}$ & $\mathrm{U}^{22}$ & $\mathrm{U}^{33}$ & $\mathrm{U}^{23}$ & $\mathrm{U}^{13}$ & $\mathrm{U}^{12}$ \\
\hline $\mathrm{C}(1)$ & $21(2)$ & $17(1)$ & $18(1)$ & $2(1)$ & $4(1)$ & $-1(1)$ \\
$\mathrm{C}(2)$ & $24(2)$ & $18(2)$ & $20(1)$ & $-2(1)$ & $-9(1)$ & $4(1)$ \\
$\mathrm{C}(3)$ & $18(2)$ & $16(2)$ & $19(1)$ & $1(1)$ & $-3(1)$ & $2(1)$ \\
$\mathrm{C}(4)$ & $15(1)$ & $22(2)$ & $22(1)$ & $-3(1)$ & $-1(1)$ & $3(1)$ \\
$\mathrm{C}(5)$ & $21(2)$ & $28(2)$ & $14(1)$ & $0(1)$ & $2(1)$ & $3(1)$ \\
$\mathrm{C}(6)$ & $25(2)$ & $21(2)$ & $14(1)$ & $-4(1)$ & $-3(1)$ & $1(1)$ \\
$\mathrm{C}(7)$ & $19(1)$ & $18(2)$ & $21(1)$ & $-1(1)$ & $-3(1)$ & $-1(1)$ \\
$\mathrm{C}(8)$ & $17(2)$ & $18(2)$ & $17(1)$ & $2(1)$ & $-3(1)$ & $3(1)$ \\
$\mathrm{C}(9)$ & $22(2)$ & $21(2)$ & $20(1)$ & $1(1)$ & $1(1)$ & $-2(1)$ \\
$\mathrm{C}(10)$ & $17(2)$ & $34(2)$ & $28(2)$ & $0(1)$ & $-6(1)$ & $-4(1)$ \\
$\mathrm{C}(11)$ & $19(2)$ & $26(2)$ & $29(2)$ & $-6(1)$ & $-8(1)$ & $5(1)$ \\
$\mathrm{C}(12)$ & $33(2)$ & $23(2)$ & $26(1)$ & $2(1)$ & $-8(1)$ & $3(1)$ \\
$\mathrm{O}(1)$ & $20(1)$ & $19(1)$ & $21(1)$ & $-3(1)$ & $-5(1)$ & $4(1)$ \\
$\mathrm{O}(2)$ & $27(1)$ & $22(1)$ & $19(1)$ & $-4(1)$ & $-5(1)$ & $8(1)$ \\
$\mathrm{O}(3)$ & $17(1)$ & $22(1)$ & $15(1)$ & $-2(1)$ & $0(1)$ & $4(1)$ \\
$\mathrm{O}(4)$ & $25(1)$ & $20(1)$ & $27(1)$ & $0(1)$ & $-3(1)$ & $8(1)$ \\
$\mathrm{O}(5)$ & $25(1)$ & $29(1)$ & $16(1)$ & $2(1)$ & $-2(1)$ & $-5(1)$ \\
& & & & & & \\
\hline & & & & & &
\end{tabular}


Table 5. Hydrogen coordinates ( $\left.\times 10^{4}\right)$ and isotropic displacement parameters $\left(\AA^{2} \times 10^{3}\right)$ for ent-17.

\begin{tabular}{|c|c|c|c|c|}
\hline & $\mathrm{x}$ & $\mathrm{y}$ & $\mathrm{z}$ & $\mathrm{U}(\mathrm{eq})$ \\
\hline $\mathrm{H}(2 \mathrm{~A})$ & 7293 & 911 & 1312 & 25 \\
\hline $\mathrm{H}(2 \mathrm{~B})$ & 8249 & 2519 & 533 & 25 \\
\hline $\mathrm{H}(3)$ & 7535 & 2701 & 3184 & 21 \\
\hline $\mathrm{H}(5)$ & 5656 & 7210 & 4344 & 25 \\
\hline $\mathrm{H}(6 \mathrm{~A})$ & 7926 & 7934 & 3012 & 24 \\
\hline $\mathrm{H}(6 \mathrm{~B})$ & 8112 & 8219 & 4527 & 24 \\
\hline $\mathrm{H}(7 \mathrm{~A})$ & 10312 & 6606 & 3960 & 23 \\
\hline $\mathrm{H}(7 \mathrm{~B})$ & 9302 & 4961 & 4651 & 23 \\
\hline $\mathrm{H}(9 \mathrm{~A})$ & 11090 & 6162 & 1825 & 31 \\
\hline $\mathrm{H}(9 \mathrm{~B})$ & 9362 & 6553 & 1271 & 31 \\
\hline $\mathrm{H}(9 \mathrm{C})$ & 10265 & 4576 & 882 & 31 \\
\hline $\mathrm{H}(10 \mathrm{~A})$ & 3910 & 3504 & 2111 & 40 \\
\hline $\mathrm{H}(10 \mathrm{~B})$ & 3715 & 4252 & 3549 & 40 \\
\hline $\mathrm{H}(10 \mathrm{C})$ & 4982 & 2595 & 3249 & 40 \\
\hline $\mathrm{H}(11)$ & 4051 & 7771 & 2424 & 30 \\
\hline $\mathrm{H}(12 \mathrm{~A})$ & 6102 & 7265 & 463 & 33 \\
\hline $\mathrm{H}(12 \mathrm{~B})$ & 4713 & 8935 & 531 & 33 \\
\hline $\mathrm{H}(5 \mathrm{~A})$ & 7033 & 4934 & 5542 & 35 \\
\hline
\end{tabular}


Table 6. Torsion angles $\left[{ }^{\circ}\right]$ for ent-17.

\begin{tabular}{|c|c|}
\hline $\mathrm{O}(2)-\mathrm{C}(2)-\mathrm{C}(3)-\mathrm{O}(3)$ & $163.6(2)$ \\
\hline $\mathrm{O}(2)-\mathrm{C}(2)-\mathrm{C}(3)-\mathrm{C}(8)$ & $45.4(3)$ \\
\hline $\mathrm{O}(3)-\mathrm{C}(4)-\mathrm{C}(5)-\mathrm{O}(5)$ & $-89.4(3)$ \\
\hline $\mathrm{C}(11)-\mathrm{C}(4)-\mathrm{C}(5)-\mathrm{O}(5)$ & $153.9(2)$ \\
\hline $\mathrm{C}(10)-\mathrm{C}(4)-\mathrm{C}(5)-\mathrm{O}(5)$ & $33.4(3)$ \\
\hline $\mathrm{O}(3)-\mathrm{C}(4)-\mathrm{C}(5)-\mathrm{C}(6)$ & $36.1(3)$ \\
\hline $\mathrm{C}(11)-\mathrm{C}(4)-\mathrm{C}(5)-\mathrm{C}(6)$ & $-80.6(3)$ \\
\hline $\mathrm{C}(10)-\mathrm{C}(4)-\mathrm{C}(5)-\mathrm{C}(6)$ & $158.9(3)$ \\
\hline $\mathrm{O}(5)-\mathrm{C}(5)-\mathrm{C}(6)-\mathrm{C}(7)$ & $37.5(3)$ \\
\hline $\mathrm{C}(4)-\mathrm{C}(5)-\mathrm{C}(6)-\mathrm{C}(7)$ & $-86.3(3)$ \\
\hline $\mathrm{C}(5)-\mathrm{C}(6)-\mathrm{C}(7)-\mathrm{C}(8)$ & $70.8(3)$ \\
\hline $\mathrm{C}(6)-\mathrm{C}(7)-\mathrm{C}(8)-\mathrm{O}(1)$ & $-164.4(2)$ \\
\hline $\mathrm{C}(6)-\mathrm{C}(7)-\mathrm{C}(8)-\mathrm{C}(9)$ & $78.6(3)$ \\
\hline $\mathrm{C}(6)-\mathrm{C}(7)-\mathrm{C}(8)-\mathrm{C}(3)$ & $-50.7(3)$ \\
\hline $\mathrm{O}(3)-\mathrm{C}(3)-\mathrm{C}(8)-\mathrm{O}(1)$ & $-174.28(19)$ \\
\hline $\mathrm{C}(2)-\mathrm{C}(3)-\mathrm{C}(8)-\mathrm{O}(1)$ & $-59.0(3)$ \\
\hline $\mathrm{O}(3)-\mathrm{C}(3)-\mathrm{C}(8)-\mathrm{C}(9)$ & $-55.8(3)$ \\
\hline $\mathrm{C}(2)-\mathrm{C}(3)-\mathrm{C}(8)-\mathrm{C}(9)$ & $59.5(3)$ \\
\hline $\mathrm{O}(3)-\mathrm{C}(3)-\mathrm{C}(8)-\mathrm{C}(7)$ & $73.0(3)$ \\
\hline $\mathrm{C}(2)-\mathrm{C}(3)-\mathrm{C}(8)-\mathrm{C}(7)$ & $-171.8(2)$ \\
\hline $\mathrm{O}(3)-\mathrm{C}(4)-\mathrm{C}(11)-\mathrm{C}(12)$ & $-3.5(4)$ \\
\hline$C(10)-C(4)-C(11)-C(12)$ & $-120.0(3)$ \\
\hline $\mathrm{C}(5)-\mathrm{C}(4)-\mathrm{C}(11)-\mathrm{C}(12)$ & $117.7(3)$ \\
\hline $\mathrm{O}(4)-\mathrm{C}(1)-\mathrm{O}(1)-\mathrm{C}(8)$ & $170.5(2)$ \\
\hline $\mathrm{O}(2)-\mathrm{C}(1)-\mathrm{O}(1)-\mathrm{C}(8)$ & $-9.8(4)$ \\
\hline $\mathrm{C}(9)-\mathrm{C}(8)-\mathrm{O}(1)-\mathrm{C}(1)$ & $-78.5(3)$ \\
\hline $\mathrm{C}(7)-\mathrm{C}(8)-\mathrm{O}(1)-\mathrm{C}(1)$ & $161.8(2)$ \\
\hline $\mathrm{C}(3)-\mathrm{C}(8)-\mathrm{O}(1)-\mathrm{C}(1)$ & $43.4(3)$ \\
\hline $\mathrm{O}(4)-\mathrm{C}(1)-\mathrm{O}(2)-\mathrm{C}(2)$ & $171.3(2)$ \\
\hline $\mathrm{O}(1)-\mathrm{C}(1)-\mathrm{O}(2)-\mathrm{C}(2)$ & $-8.4(4)$ \\
\hline $\mathrm{C}(3)-\mathrm{C}(2)-\mathrm{O}(2)-\mathrm{C}(1)$ & $-11.4(4)$ \\
\hline $\mathrm{C}(2)-\mathrm{C}(3)-\mathrm{O}(3)-\mathrm{C}(4)$ & $142.0(2)$ \\
\hline $\mathrm{C}(8)-\mathrm{C}(3)-\mathrm{O}(3)-\mathrm{C}(4)$ & $-100.2(2)$ \\
\hline $\mathrm{C}(11)-\mathrm{C}(4)-\mathrm{O}(3)-\mathrm{C}(3)$ & $166.9(2)$ \\
\hline $\mathrm{C}(10)-\mathrm{C}(4)-\mathrm{O}(3)-\mathrm{C}(3)$ & $-76.5(3)$ \\
\hline $\mathrm{C}(5)-\mathrm{C}(4)-\mathrm{O}(3)-\mathrm{C}(3)$ & $48.0(3)$ \\
\hline
\end{tabular}

Symmetry transformations used to generate equivalent atoms: 
Thermal ellipsoid diagram for ent-17:

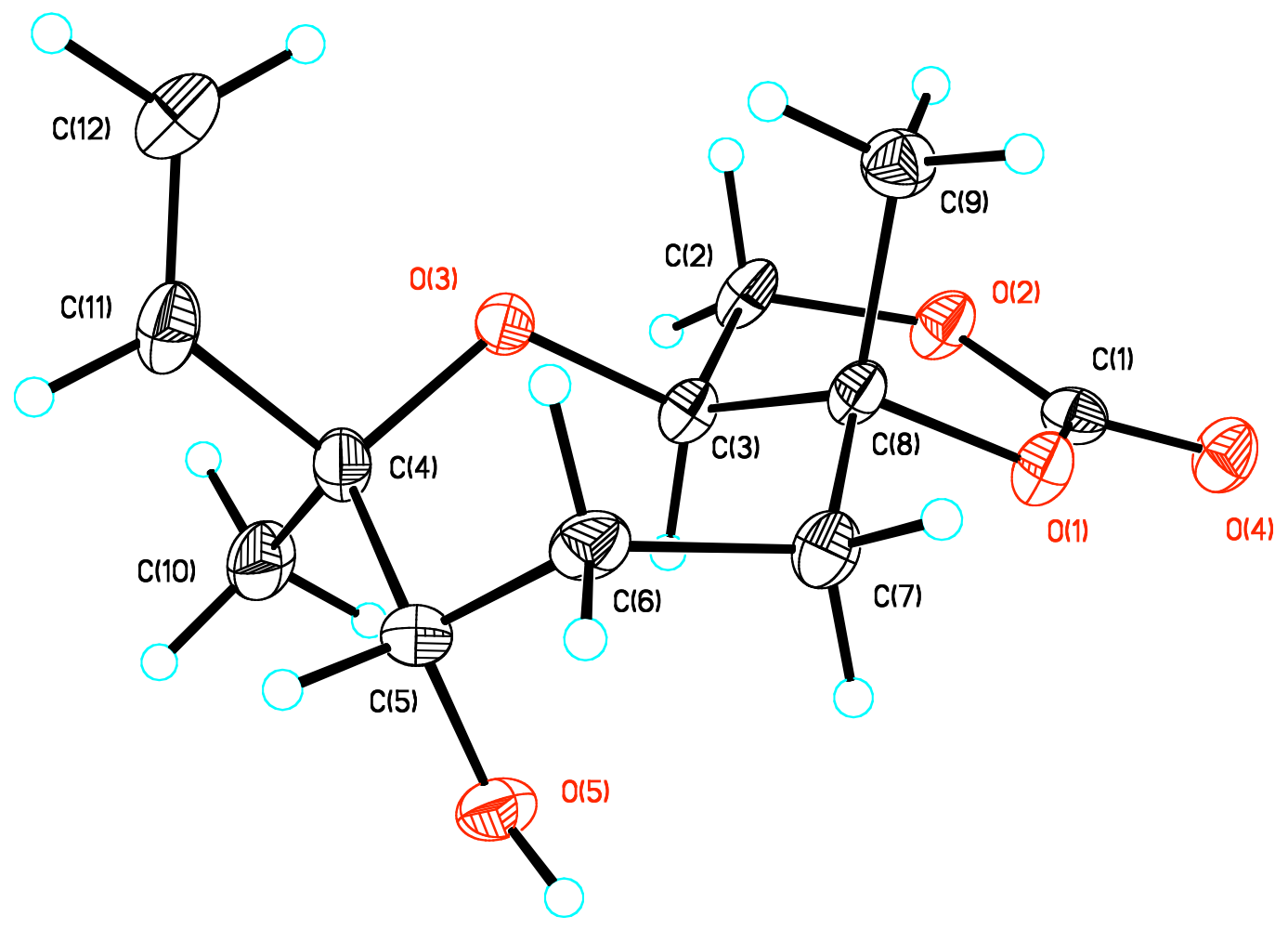




\section{Tricyclic 18.}

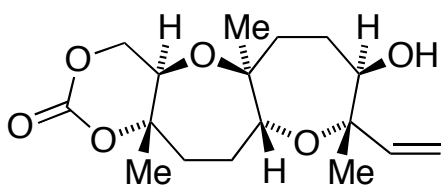

18

The triepoxide 16 (63 mg, $0.16 \mathrm{mmol})$ was dissolved in anhydrous dichloromethane $(3.9 \mathrm{~mL})$, and ytterbium (III) triflate $(600 \mathrm{mg}, 0.97 \mathrm{mmol}$ ) was added in one portion at room temperature. The reaction was stirred for 2 hours, and then $10 \mathrm{~mL}$ of $\mathrm{NaHCO}_{3}$ were added. The mixture was poured into water and extracted with $\mathrm{CH}_{2} \mathrm{Cl}_{2}$. The organic phases were combined, dried over $\mathrm{MgSO}_{4}$, and the solvent was removed under reduced pressure. Compound 18 (30 mg, 56\%) was isolated after purification by column chromatography (hexanes:ethyl acetate, increasing gradually the polarity from $3: 1$ to $1: 1$ to $1: 3)$.

m.p. $>50^{\circ} \mathrm{C}$ (dec.).

$[\alpha]_{\mathrm{D}}^{25}=+14.6\left(c 0.555, \mathrm{CHCl}_{3}\right)$.

El. anal. calcd. for $\mathrm{C}_{17} \mathrm{H}_{26} \mathrm{O}_{6}$ : C, 62.56; H, 8.03. Found: C, 62.06; H, 8.23.

IR (neat): 3478 (br), 3092, 3058, 2980, 2933, 2878, 1746, 1642, 1486, 1454, 1405, 1384, 1364, $1272,1253,1216,1102,1077,1031,998,927,866,772,736,706 \mathrm{~cm}^{-1}$.

${ }^{1} \mathrm{H}$ NMR $\left(600 \mathrm{MHz}, \mathrm{CDCl}_{3}\right) \delta 5.66(\mathrm{dd}, J=17.1,10.5 \mathrm{~Hz}, 1 \mathrm{H}), 5.25(\mathrm{dd}, J=17.1,1.5 \mathrm{~Hz}, 1 \mathrm{H})$, $5.04(\mathrm{dd}, J=10.5,1.5 \mathrm{~Hz}, 1 \mathrm{H}), 4.18(\mathrm{dd}, J=9.6,6.0 \mathrm{~Hz}, 1 \mathrm{H}), 4.14-4.10(\mathrm{~m}, 1 \mathrm{H}), 4.06$ (t, $J=9.9$ $\mathrm{Hz}, 1 \mathrm{H}), 3.84$ (d, $J=6.6 \mathrm{~Hz}, 1 \mathrm{H}), 3.80$ (dd, $J=11.4,2.4 \mathrm{~Hz}, 1 \mathrm{H}), 2.17$ (ddd, $J=15.6,5.4,2.4$ $\mathrm{Hz}, 1 \mathrm{H}), 2.06-1.94(\mathrm{~m}, 2 \mathrm{H}), 1.82(\mathrm{dd}, J=13.5,2.4 \mathrm{~Hz}, 1 \mathrm{H}), 1.73-1.65(\mathrm{~m}, 2 \mathrm{H}), 1.53$ (dquint, $J=$ 15.0, $2.7 \mathrm{~Hz}, 1 \mathrm{H}), 1.48-1.42(\mathrm{~m}, 1 \mathrm{H}), 1.43$ (s, 3H), 1.30 (s, 3H), 1.27 (s, 3H).

${ }^{13} \mathrm{C}$ NMR $(150.8 \mathrm{MHz}) \delta 149.1,142.9,113.3,83.2,80.9,80.2,77.5,74.8,66.9,63.6,37.2,34.7$, $28.0,24.4,22.1,20.5,16.5$. 


\section{Formation of bicyclic bromooxepanes 19 and 20:}

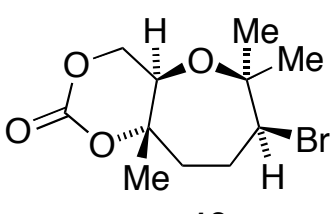

19

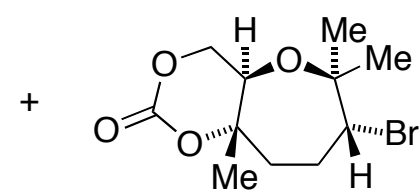

20

The Boc-protected geraniol monoepoxide 11 (600 mg, $2.22 \mathrm{mmol})$ was dissolved in anhydrous dichloromethane $(44 \mathrm{~mL})$, and bromonium bis-sym-collidine perchlorate $(2.80 \mathrm{~g}, 6.64 \mathrm{mmol})$ was added in one portion at $-40^{\circ} \mathrm{C}$. The reaction was stirred for $15 \mathrm{~min}$., and then it was poured into water and a few milliliters of cyclohexene were added. The layers were separated and the aqueous phase was extracted with more dichloromethane. The organic fractions were combined, dried over magnesium sulfate and then filtered. The solvent was evaporated under reduced pressure, and the crude was finally purified by column chromatography (hexanes:ethyl acetate, increasing gradually the polarity from $3: 1$ to $1: 1$ ) to give the mixture of diastereomeric oxepanes (325 mg, 50\%). The mixture of diastereomers 19 and $\mathbf{2 0}$ could be separated with careful purification on silica gel (hexanes: ethyl acetate 4:1).

The higher $\mathrm{R}_{\mathrm{f}}$ product was determined to be diastereomer 19:

IR (neat): 2981, 2940, 2877, 1755, 1490, 1464, 1410, 1390, 1369, 1271, 1246, 1232, 1173, 1138 , $1104,1069,982,909,764,730 \mathrm{~cm}^{-1}$.

${ }^{1} \mathrm{H}$ NMR $\left(400 \mathrm{MHz}, \mathrm{CDCl}_{3}\right) \delta 4.20(\mathrm{dd}, J=10.8,6.8 \mathrm{~Hz}, 1 \mathrm{H}), 4.09-4.02(\mathrm{~m}, 2 \mathrm{H}), 3.86(\mathrm{dd}, J=$ $10.4,6.4 \mathrm{~Hz}, 1 \mathrm{H}), 2.24-2.10(\mathrm{~m}, 2 \mathrm{H}), 2.02(\mathrm{ddd}, J=13.9,5.3,2.6 \mathrm{~Hz}, 1 \mathrm{H}), 1.79$ (approx. td, $J=$ 12.9, 3.7 Hz, 2H), 1.43 (s, 3H), 1.38 (s, 3H), 1.37 (s, 3H).

${ }^{13} \mathrm{C}$ NMR $(100.5 \mathrm{MHz}) \delta 147.5,82.4,79.5,66.5,64.0,57.5,41.6,30.2,27.7,25.3,24.6,19.1$.

The lower $\mathrm{R}_{\mathrm{f}}$ product was determined to be diastereomer $\mathbf{2 0}$ :

$[\alpha]_{\mathrm{D}}^{25}=+2.0\left(c 1.935, \mathrm{CHCl}_{3}\right)$.

El. anal. calcd. for $\mathrm{C}_{11} \mathrm{H}_{17} \mathrm{BrO}_{4}$ : C, 45.07; H, 5.85; Br, 27.26. Found: C, 44.77; H, 6.10; Br, 29.57.

IR (neat): 2980, 2938, 2875, 1756, 1482, 1461, 1405, 1386, 1355, 1253, 1229, 1215, 1128, 1102 , $1065,980,902,766,730 \mathrm{~cm}^{-1}$.

${ }^{1} \mathrm{H}$ NMR (400 MHz, $\left.\mathrm{CDCl}_{3}\right) \delta 4.47(\mathrm{dd}, J=6.2,2.6 \mathrm{~Hz}, 1 \mathrm{H}), 4.33(\mathrm{dd}, J=10.8,6.4 \mathrm{~Hz}, 1 \mathrm{H})$, 4.19 (dd, $J=10.8,6.4 \mathrm{~Hz}, 1 \mathrm{H}), 4.01$ (t, $J=10.8 \mathrm{~Hz}, 1 \mathrm{H}), 2.54-2.44$ (m, 1H), 2.20-2.00 (m, 2H), 1.92 (ddd, $J=14.0,5.4,3.2 \mathrm{~Hz}, 1 \mathrm{H}), 1.46$ (d, $J=0.8 \mathrm{~Hz}, 3 \mathrm{H}), 1.43$ (s, 3H), 1.32 (s, 3H).

${ }^{13} \mathrm{C}$ NMR (100.5 MHz) $\delta 149.2,83.0,78.4,66.6,63.8,63.2,36.9,29.6,27.5,27.4,24.6,19.8$. 
Table 1. Crystal data and structure refinement for 19.

Identification code

Empirical formula

Formula weight

Temperature

Wavelength

Crystal system

Space group

Unit cell dimensions

Volume

Z

Density (calculated)

Absorption coefficient

$\mathrm{F}(000)$

Crystal size

Theta range for data collection

Index ranges

Reflections collected

Independent reflections

Completeness to theta $=66.77^{\circ}$

Absorption correction

Max. and min. transmission

Refinement method

Data / restraints / parameters

Goodness-of-fit on $\mathrm{F}^{2}$

Final R indices [I $>2 \operatorname{sigma}(\mathrm{I})]$

$\mathrm{R}$ indices (all data)

Absolute structure parameter

Largest diff. peak and hole $\mathrm{fb} 21 \mathrm{as}$

C11 H17 Br O4

293.16

100(2) K

$1.54178 \AA$

Trigonal

P3(2)21

$\mathrm{a}=7.2078(1) \AA$

$\alpha=90^{\circ}$.

$\mathrm{b}=7.2078(1) \AA$

$\beta=90^{\circ}$.

$\mathrm{c}=42.0575(5) \AA$

$\gamma=120^{\circ}$.

6

$1.544 \mathrm{Mg} / \mathrm{m}^{3}$

$4.444 \mathrm{~mm}^{-1}$

900

$0.31 \times 0.21 \times 0.10 \mathrm{~mm}^{3}$

3.15 to $66.77^{\circ}$.

$-7<=\mathrm{h}<=8,-8<=\mathrm{k}<=7,-49<=\mathrm{l}<=42$

8437

$2103[\mathrm{R}(\mathrm{int})=0.0199]$

$97.4 \%$

Semi-empirical from equivalents

1.000 and 0.701792

Full-matrix least-squares on $\mathrm{F}^{2}$

$2103 / 0 / 191$

1.152

$\mathrm{R} 1=0.0251, \mathrm{wR} 2=0.0857$

$\mathrm{R} 1=0.0270, \mathrm{wR} 2=0.0979$

$0.05(3)$

0.580 and -0.580 e. $\AA^{-3}$ 
Table 2. Atomic coordinates $\left(\times 10^{4}\right)$ and equivalent isotropic displacement parameters $\left(\AA^{2} \times 10^{3}\right)$ for 19. $U(e q)$ is defined as one third of the trace of the orthogonalized $U^{i j}$ tensor.

\begin{tabular}{lrrrr}
\hline & $\mathrm{x}$ & $\mathrm{y}$ & $\mathrm{z}$ & $\mathrm{U}(\mathrm{eq})$ \\
\hline $\mathrm{Br}(1)$ & $46(1)$ & $8041(1)$ & $293(1)$ & $31(1)$ \\
$\mathrm{C}(1)$ & $1392(5)$ & $7241(5)$ & $637(1)$ & $20(1)$ \\
$\mathrm{C}(2)$ & $1578(6)$ & $5291(6)$ & $530(1)$ & $22(1)$ \\
$\mathrm{C}(3)$ & $2849(5)$ & $5275(6)$ & $1071(1)$ & $18(1)$ \\
$\mathrm{C}(4)$ & $3210(6)$ & $3597(6)$ & $1225(1)$ & $23(1)$ \\
$\mathrm{C}(5)$ & $3889(6)$ & $5701(6)$ & $1710(1)$ & $21(1)$ \\
$\mathrm{C}(6)$ & $4529(6)$ & $7487(5)$ & $1188(1)$ & $21(1)$ \\
$\mathrm{C}(7)$ & $3941(6)$ & $9181(6)$ & $1099(1)$ & $24(1)$ \\
$\mathrm{C}(8)$ & $3488(7)$ & $9233(6)$ & $741(1)$ & $25(1)$ \\
$\mathrm{C}(9)$ & $-585(6)$ & $3258(6)$ & $554(1)$ & $25(1)$ \\
$\mathrm{C}(10)$ & $2565(6)$ & $5541(6)$ & $204(1)$ & $26(1)$ \\
$\mathrm{C}(11)$ & $6814(6)$ & $8146(7)$ & $1099(1)$ & $31(1)$ \\
$\mathrm{O}(1)$ & $3104(4)$ & $5124(4)$ & $739(1)$ & $21(1)$ \\
$\mathrm{O}(2)$ & $3330(5)$ & $3842(4)$ & $1568(1)$ & $32(1)$ \\
$\mathrm{O}(3)$ & $3923(4)$ & $5785(4)$ & $1997(1)$ & $25(1)$ \\
$\mathrm{O}(4)$ & $4407(4)$ & $7427(4)$ & $1540(1)$ & $26(1)$ \\
& & & & \\
\hline & & & &
\end{tabular}


Table 3. Bond lengths $[\AA]$ and angles $\left[{ }^{\circ}\right]$ for 19.

\begin{tabular}{|c|c|c|c|}
\hline $\operatorname{Br}(1)-C(1)$ & $1.981(3)$ & & \\
\hline $\mathrm{C}(1)-\mathrm{C}(8)$ & $1.538(5)$ & $\mathrm{C}(8)-\mathrm{C}(1)-\mathrm{C}(2)$ & $116.1(3)$ \\
\hline$C(1)-C(2)$ & $1.544(5)$ & $\mathrm{C}(8)-\mathrm{C}(1)-\mathrm{Br}(1)$ & $109.3(2)$ \\
\hline $\mathrm{C}(1)-\mathrm{H}(1)$ & $0.98(3)$ & $C(2)-C(1)-B r(1)$ & $109.4(2)$ \\
\hline $\mathrm{C}(2)-\mathrm{O}(1)$ & $1.457(4)$ & $\mathrm{C}(8)-\mathrm{C}(1)-\mathrm{H}(1)$ & 109.9(19) \\
\hline$C(2)-C(10)$ & $1.516(4)$ & $\mathrm{C}(2)-\mathrm{C}(1)-\mathrm{H}(1)$ & $113(2)$ \\
\hline$C(2)-C(9)$ & $1.518(5)$ & $\mathrm{Br}(1)-\mathrm{C}(1)-\mathrm{H}(1)$ & $97(2)$ \\
\hline $\mathrm{C}(3)-\mathrm{O}(1)$ & $1.421(4)$ & $\mathrm{O}(1)-\mathrm{C}(2)-\mathrm{C}(10)$ & $103.1(3)$ \\
\hline $\mathrm{C}(3)-\mathrm{C}(4)$ & $1.505(5)$ & $\mathrm{O}(1)-\mathrm{C}(2)-\mathrm{C}(9)$ & $109.5(3)$ \\
\hline$C(3)-C(6)$ & $1.523(5)$ & $\mathrm{C}(10)-\mathrm{C}(2)-\mathrm{C}(9)$ & $111.7(3)$ \\
\hline $\mathrm{C}(3)-\mathrm{H}(3)$ & $0.97(4)$ & $\mathrm{O}(1)-\mathrm{C}(2)-\mathrm{C}(1)$ & $108.3(3)$ \\
\hline $\mathrm{C}(4)-\mathrm{O}(2)$ & $1.451(4)$ & $\mathrm{C}(10)-\mathrm{C}(2)-\mathrm{C}(1)$ & $113.8(3)$ \\
\hline $\mathrm{C}(4)-\mathrm{H}(4 \mathrm{~A})$ & $0.93(4)$ & $\mathrm{C}(9)-\mathrm{C}(2)-\mathrm{C}(1)$ & $110.2(3)$ \\
\hline $\mathrm{C}(4)-\mathrm{H}(4 \mathrm{~B})$ & $1.01(4)$ & $\mathrm{O}(1)-\mathrm{C}(3)-\mathrm{C}(4)$ & $106.3(3)$ \\
\hline $\mathrm{C}(5)-\mathrm{O}(3)$ & $1.209(4)$ & $\mathrm{O}(1)-\mathrm{C}(3)-\mathrm{C}(6)$ & $109.4(3)$ \\
\hline $\mathrm{C}(5)-\mathrm{O}(4)$ & $1.317(4)$ & $C(4)-C(3)-C(6)$ & 109.6(3) \\
\hline $\mathrm{C}(5)-\mathrm{O}(2)$ & $1.332(4)$ & $\mathrm{O}(1)-\mathrm{C}(3)-\mathrm{H}(3)$ & $112(2)$ \\
\hline $\mathrm{C}(6)-\mathrm{O}(4)$ & $1.482(4)$ & $\mathrm{C}(4)-\mathrm{C}(3)-\mathrm{H}(3)$ & $111(2)$ \\
\hline$C(6)-C(11)$ & $1.515(5)$ & $\mathrm{C}(6)-\mathrm{C}(3)-\mathrm{H}(3)$ & $108(2)$ \\
\hline$C(6)-C(7)$ & $1.526(5)$ & $\mathrm{O}(2)-\mathrm{C}(4)-\mathrm{C}(3)$ & $110.7(3)$ \\
\hline $\mathrm{C}(7)-\mathrm{C}(8)$ & $1.547(5)$ & $\mathrm{O}(2)-\mathrm{C}(4)-\mathrm{H}(4 \mathrm{~A})$ & $107(2)$ \\
\hline $\mathrm{C}(7)-\mathrm{H}(7 \mathrm{~A})$ & $1.00(4)$ & $\mathrm{C}(3)-\mathrm{C}(4)-\mathrm{H}(4 \mathrm{~A})$ & $111(2)$ \\
\hline $\mathrm{C}(7)-\mathrm{H}(7 \mathrm{~B})$ & $1.02(4)$ & $\mathrm{O}(2)-\mathrm{C}(4)-\mathrm{H}(4 \mathrm{~B})$ & $103(2)$ \\
\hline $\mathrm{C}(8)-\mathrm{H}(8 \mathrm{~A})$ & $1.05(3)$ & $\mathrm{C}(3)-\mathrm{C}(4)-\mathrm{H}(4 \mathrm{~B})$ & $110(2)$ \\
\hline $\mathrm{C}(8)-\mathrm{H}(8 \mathrm{~B})$ & $0.94(4)$ & $\mathrm{H}(4 \mathrm{~A})-\mathrm{C}(4)-\mathrm{H}(4 \mathrm{~B})$ & $115(3)$ \\
\hline $\mathrm{C}(9)-\mathrm{H}(9 \mathrm{~A})$ & 0.9800 & $\mathrm{O}(3)-\mathrm{C}(5)-\mathrm{O}(4)$ & $120.4(3)$ \\
\hline $\mathrm{C}(9)-\mathrm{H}(9 \mathrm{~B})$ & 0.9800 & $\mathrm{O}(3)-\mathrm{C}(5)-\mathrm{O}(2)$ & 119.1(3) \\
\hline C(9)-H(9C) & 0.9800 & $\mathrm{O}(4)-\mathrm{C}(5)-\mathrm{O}(2)$ & $120.5(3)$ \\
\hline $\mathrm{C}(10)-\mathrm{H}(10 \mathrm{~A})$ & 0.9800 & $\mathrm{O}(4)-\mathrm{C}(6)-\mathrm{C}(11)$ & 107.2(3) \\
\hline $\mathrm{C}(10)-\mathrm{H}(10 \mathrm{~B})$ & 0.9800 & $\mathrm{O}(4)-\mathrm{C}(6)-\mathrm{C}(3)$ & $106.8(3)$ \\
\hline $\mathrm{C}(10)-\mathrm{H}(10 \mathrm{C})$ & 0.9800 & $\mathrm{C}(11)-\mathrm{C}(6)-\mathrm{C}(3)$ & $114.8(3)$ \\
\hline $\mathrm{C}(11)-\mathrm{H}(11 \mathrm{~A})$ & $0.96(5)$ & $\mathrm{O}(4)-\mathrm{C}(6)-\mathrm{C}(7)$ & $103.4(3)$ \\
\hline $\mathrm{C}(11)-\mathrm{H}(11 \mathrm{~B})$ & $0.96(4)$ & $C(11)-C(6)-C(7)$ & $112.4(3)$ \\
\hline $\mathrm{C}(11)-\mathrm{H}(11 \mathrm{C})$ & $0.94(5)$ & $C(3)-C(6)-C(7)$ & $111.4(3)$ \\
\hline
\end{tabular}




\begin{tabular}{|c|c|}
\hline$C(6)-C(7)-C(8)$ & $113.9(3)$ \\
\hline $\mathrm{C}(6)-\mathrm{C}(7)-\mathrm{H}(7 \mathrm{~A})$ & $111(2)$ \\
\hline $\mathrm{C}(8)-\mathrm{C}(7)-\mathrm{H}(7 \mathrm{~A})$ & $108(2)$ \\
\hline $\mathrm{C}(6)-\mathrm{C}(7)-\mathrm{H}(7 \mathrm{~B})$ & $105(2)$ \\
\hline $\mathrm{C}(8)-\mathrm{C}(7)-\mathrm{H}(7 \mathrm{~B})$ & $107(2)$ \\
\hline $\mathrm{H}(7 \mathrm{~A})-\mathrm{C}(7)-\mathrm{H}(7 \mathrm{~B})$ & $111(3)$ \\
\hline $\mathrm{C}(1)-\mathrm{C}(8)-\mathrm{C}(7)$ & $112.0(3)$ \\
\hline $\mathrm{C}(1)-\mathrm{C}(8)-\mathrm{H}(8 \mathrm{~A})$ & 111.2(19) \\
\hline $\mathrm{C}(7)-\mathrm{C}(8)-\mathrm{H}(8 \mathrm{~A})$ & $112.4(19)$ \\
\hline $\mathrm{C}(1)-\mathrm{C}(8)-\mathrm{H}(8 \mathrm{~B})$ & $108(2)$ \\
\hline $\mathrm{C}(7)-\mathrm{C}(8)-\mathrm{H}(8 \mathrm{~B})$ & $106(2)$ \\
\hline $\mathrm{H}(8 \mathrm{~A})-\mathrm{C}(8)-\mathrm{H}(8 \mathrm{~B})$ & $107(3)$ \\
\hline $\mathrm{C}(2)-\mathrm{C}(9)-\mathrm{H}(9 \mathrm{~A})$ & 109.5 \\
\hline $\mathrm{C}(2)-\mathrm{C}(9)-\mathrm{H}(9 \mathrm{~B})$ & 109.5 \\
\hline $\mathrm{H}(9 \mathrm{~A})-\mathrm{C}(9)-\mathrm{H}(9 \mathrm{~B})$ & 109.5 \\
\hline $\mathrm{C}(2)-\mathrm{C}(9)-\mathrm{H}(9 \mathrm{C})$ & 109.5 \\
\hline $\mathrm{H}(9 \mathrm{~A})-\mathrm{C}(9)-\mathrm{H}(9 \mathrm{C})$ & 109.5 \\
\hline $\mathrm{H}(9 \mathrm{~B})-\mathrm{C}(9)-\mathrm{H}(9 \mathrm{C})$ & 109.5 \\
\hline $\mathrm{C}(2)-\mathrm{C}(10)-\mathrm{H}(10 \mathrm{~A})$ & 109.5 \\
\hline $\mathrm{C}(2)-\mathrm{C}(10)-\mathrm{H}(10 \mathrm{~B})$ & 109.5 \\
\hline $\mathrm{H}(10 \mathrm{~A})-\mathrm{C}(10)-\mathrm{H}(10 \mathrm{~B})$ & 109.5 \\
\hline $\mathrm{C}(2)-\mathrm{C}(10)-\mathrm{H}(10 \mathrm{C})$ & 109.5 \\
\hline $\mathrm{H}(10 \mathrm{~A})-\mathrm{C}(10)-\mathrm{H}(10 \mathrm{C})$ & 109.5 \\
\hline $\mathrm{H}(10 \mathrm{~B})-\mathrm{C}(10)-\mathrm{H}(10 \mathrm{C})$ & 109.5 \\
\hline $\mathrm{C}(6)-\mathrm{C}(11)-\mathrm{H}(11 \mathrm{~A})$ & $112(3)$ \\
\hline $\mathrm{C}(6)-\mathrm{C}(11)-\mathrm{H}(11 \mathrm{~B})$ & $107(3)$ \\
\hline $\mathrm{H}(11 \mathrm{~A})-\mathrm{C}(11)-\mathrm{H}(11 \mathrm{~B})$ & $113(4)$ \\
\hline $\mathrm{C}(6)-\mathrm{C}(11)-\mathrm{H}(11 \mathrm{C})$ & $108(3)$ \\
\hline $\mathrm{H}(11 \mathrm{~A})-\mathrm{C}(11)-\mathrm{H}(11 \mathrm{C})$ & $108(4)$ \\
\hline $\mathrm{H}(11 \mathrm{~B})-\mathrm{C}(11)-\mathrm{H}(11 \mathrm{C})$ & $108(4)$ \\
\hline $\mathrm{C}(3)-\mathrm{O}(1)-\mathrm{C}(2)$ & $117.0(2)$ \\
\hline $\mathrm{C}(5)-\mathrm{O}(2)-\mathrm{C}(4)$ & $122.5(3)$ \\
\hline $\mathrm{C}(5)-\mathrm{O}(4)-\mathrm{C}(6)$ & $123.6(3)$ \\
\hline
\end{tabular}

Symmetry transformations used to generate equivalent atoms: 
Table 4. Anisotropic displacement parameters $\left(\AA^{2} \times 10^{3}\right)$ for 19. The anisotropic displacement factor exponent takes the form: $-2 \pi^{2}\left[h^{2} a^{* 2} U^{11}+\ldots+2 h k a^{*} b^{*} U^{12}\right]$

\begin{tabular}{lllllll}
\hline & $\mathrm{U}^{11}$ & $\mathrm{U}^{22}$ & $\mathrm{U}^{33}$ & $\mathrm{U}^{23}$ & $\mathrm{U}^{13}$ & $\mathrm{U}^{12}$ \\
\hline $\mathrm{Br}(1)$ & $47(1)$ & $36(1)$ & $22(1)$ & $0(1)$ & $-8(1)$ & $28(1)$ \\
$\mathrm{C}(1)$ & $26(2)$ & $23(2)$ & $13(1)$ & $0(1)$ & $2(1)$ & $14(1)$ \\
$\mathrm{C}(2)$ & $23(2)$ & $29(2)$ & $13(2)$ & $2(1)$ & $4(1)$ & $13(2)$ \\
$\mathrm{C}(3)$ & $21(2)$ & $20(2)$ & $15(2)$ & $1(1)$ & $2(1)$ & $12(1)$ \\
$\mathrm{C}(4)$ & $30(2)$ & $25(2)$ & $18(2)$ & $-1(1)$ & $-2(1)$ & $18(2)$ \\
$\mathrm{C}(5)$ & $17(2)$ & $26(2)$ & $23(2)$ & $1(1)$ & $-3(1)$ & $14(1)$ \\
$\mathrm{C}(6)$ & $24(2)$ & $23(2)$ & $17(2)$ & $1(1)$ & $-3(1)$ & $13(1)$ \\
$\mathrm{C}(7)$ & $30(2)$ & $19(2)$ & $21(2)$ & $0(1)$ & $-2(2)$ & $11(2)$ \\
$\mathrm{C}(8)$ & $44(2)$ & $16(2)$ & $22(2)$ & $2(1)$ & $-4(2)$ & $19(2)$ \\
$\mathrm{C}(9)$ & $31(2)$ & $21(2)$ & $21(2)$ & $-6(1)$ & $-5(1)$ & $12(2)$ \\
$\mathrm{C}(10)$ & $33(2)$ & $29(2)$ & $19(2)$ & $1(1)$ & $9(2)$ & $16(2)$ \\
$\mathrm{C}(11)$ & $25(2)$ & $28(2)$ & $39(2)$ & $8(2)$ & $-3(2)$ & $13(2)$ \\
$\mathrm{O}(1)$ & $26(1)$ & $26(1)$ & $16(1)$ & $1(1)$ & $2(1)$ & $16(1)$ \\
$\mathrm{O}(2)$ & $57(2)$ & $25(1)$ & $18(1)$ & $2(1)$ & $-1(1)$ & $24(1)$ \\
$\mathrm{O}(3)$ & $25(1)$ & $35(1)$ & $20(1)$ & $1(1)$ & $-1(1)$ & $19(1)$ \\
$\mathrm{O}(4)$ & $38(1)$ & $23(1)$ & $18(1)$ & $-2(1)$ & $-7(1)$ & $15(1)$ \\
& & & & & & \\
\hline
\end{tabular}


Table 5. Hydrogen coordinates ( $\left.\times 10^{4}\right)$ and isotropic displacement parameters $\left(\AA^{2} \times 10^{3}\right)$ for 19.

\begin{tabular}{|c|c|c|c|c|}
\hline & $\mathrm{x}$ & $\mathrm{y}$ & $\mathrm{z}$ & $\mathrm{U}(\mathrm{eq})$ \\
\hline $\mathrm{H}(9 \mathrm{~A})$ & -443 & 2028 & 490 & 37 \\
\hline $\mathrm{H}(9 \mathrm{~B})$ & -1609 & 3370 & 414 & 37 \\
\hline $\mathrm{H}(9 \mathrm{C})$ & -1100 & 3064 & 774 & 37 \\
\hline $\mathrm{H}(10 \mathrm{~A})$ & 3893 & 6926 & 190 & 40 \\
\hline $\mathrm{H}(10 \mathrm{~B})$ & 1557 & 5473 & 41 & 40 \\
\hline $\mathrm{H}(10 \mathrm{C})$ & 2883 & 4385 & 168 & 40 \\
\hline $\mathrm{H}(1)$ & $290(50)$ & $6890(50)$ & $797(8)$ & $8(8)$ \\
\hline $\mathrm{H}(3)$ & $1440(70)$ & $5050(60)$ & $1125(9)$ & $22(10)$ \\
\hline $\mathrm{H}(4 \mathrm{~A})$ & $4500(60)$ & $3740(60)$ & 1160(9) & $21(10)$ \\
\hline $\mathrm{H}(4 \mathrm{~B})$ & $1920(60)$ & $2120(60)$ & 1193(9) & $24(10)$ \\
\hline $\mathrm{H}(7 \mathrm{~A})$ & $5100(60)$ & $10650(70)$ & 1165(8) & $16(9)$ \\
\hline $\mathrm{H}(7 \mathrm{~B})$ & $2550(70)$ & $8750(60)$ & $1218(9)$ & $24(10)$ \\
\hline $\mathrm{H}(8 \mathrm{~A})$ & $4770(60)$ & $9460(60)$ & $598(8)$ & $8(8)$ \\
\hline $\mathrm{H}(8 \mathrm{~B})$ & $3330(60)$ & $10440(60)$ & $712(7)$ & $10(8)$ \\
\hline $\mathrm{H}(11 \mathrm{~A})$ & $7230(70)$ & $7170(70)$ & 1183(10) & $34(12)$ \\
\hline $\mathrm{H}(11 \mathrm{~B})$ & $6920(70)$ & $8270(70)$ & $872(10)$ & $34(12)$ \\
\hline $\mathrm{H}(11 \mathrm{C})$ & $7730(70)$ & $9510(80)$ & $1186(11)$ & $42(13)$ \\
\hline
\end{tabular}


Table 6. Torsion angles $\left[^{\circ}\right]$ for $\mathbf{1 9}$.

\begin{tabular}{|c|c|}
\hline $\mathrm{C}(8)-\mathrm{C}(1)-\mathrm{C}(2)-\mathrm{O}(1)$ & $39.1(4)$ \\
\hline $\mathrm{Br}(1)-\mathrm{C}(1)-\mathrm{C}(2)-\mathrm{O}(1)$ & $163.4(2)$ \\
\hline$C(8)-C(1)-C(2)-C(10)$ & $-74.8(4)$ \\
\hline $\operatorname{Br}(1)-C(1)-C(2)-C(10)$ & $49.5(3)$ \\
\hline $\mathrm{C}(8)-\mathrm{C}(1)-\mathrm{C}(2)-\mathrm{C}(9)$ & $158.9(3)$ \\
\hline $\mathrm{Br}(1)-\mathrm{C}(1)-\mathrm{C}(2)-\mathrm{C}(9)$ & $-76.9(3)$ \\
\hline $\mathrm{O}(1)-\mathrm{C}(3)-\mathrm{C}(4)-\mathrm{O}(2)$ & $170.8(3)$ \\
\hline $\mathrm{C}(6)-\mathrm{C}(3)-\mathrm{C}(4)-\mathrm{O}(2)$ & $52.6(4)$ \\
\hline $\mathrm{O}(1)-\mathrm{C}(3)-\mathrm{C}(6)-\mathrm{O}(4)$ & $-171.2(3)$ \\
\hline $\mathrm{C}(4)-\mathrm{C}(3)-\mathrm{C}(6)-\mathrm{O}(4)$ & $-55.0(4)$ \\
\hline $\mathrm{O}(1)-\mathrm{C}(3)-\mathrm{C}(6)-\mathrm{C}(11)$ & $-52.5(4)$ \\
\hline$C(4)-C(3)-C(6)-C(11)$ & $63.7(4)$ \\
\hline $\mathrm{O}(1)-\mathrm{C}(3)-\mathrm{C}(6)-\mathrm{C}(7)$ & $76.6(4)$ \\
\hline $\mathrm{C}(4)-\mathrm{C}(3)-\mathrm{C}(6)-\mathrm{C}(7)$ & $-167.2(3)$ \\
\hline $\mathrm{O}(4)-\mathrm{C}(6)-\mathrm{C}(7)-\mathrm{C}(8)$ & $-168.0(3)$ \\
\hline $\mathrm{C}(11)-\mathrm{C}(6)-\mathrm{C}(7)-\mathrm{C}(8)$ & $76.7(4)$ \\
\hline$C(3)-C(6)-C(7)-C(8)$ & $-53.7(4)$ \\
\hline $\mathrm{C}(2)-\mathrm{C}(1)-\mathrm{C}(8)-\mathrm{C}(7)$ & $-88.6(4)$ \\
\hline $\mathrm{Br}(1)-\mathrm{C}(1)-\mathrm{C}(8)-\mathrm{C}(7)$ & 147.1(3) \\
\hline $\mathrm{C}(6)-\mathrm{C}(7)-\mathrm{C}(8)-\mathrm{C}(1)$ & $69.8(4)$ \\
\hline $\mathrm{C}(4)-\mathrm{C}(3)-\mathrm{O}(1)-\mathrm{C}(2)$ & $138.3(3)$ \\
\hline $\mathrm{C}(6)-\mathrm{C}(3)-\mathrm{O}(1)-\mathrm{C}(2)$ & $-103.5(3)$ \\
\hline $\mathrm{C}(10)-\mathrm{C}(2)-\mathrm{O}(1)-\mathrm{C}(3)$ & $168.5(3)$ \\
\hline $\mathrm{C}(9)-\mathrm{C}(2)-\mathrm{O}(1)-\mathrm{C}(3)$ & $-72.5(4)$ \\
\hline $\mathrm{C}(1)-\mathrm{C}(2)-\mathrm{O}(1)-\mathrm{C}(3)$ & $47.6(4)$ \\
\hline $\mathrm{O}(3)-\mathrm{C}(5)-\mathrm{O}(2)-\mathrm{C}(4)$ & $178.8(3)$ \\
\hline $\mathrm{O}(4)-\mathrm{C}(5)-\mathrm{O}(2)-\mathrm{C}(4)$ & $-1.4(5)$ \\
\hline $\mathrm{C}(3)-\mathrm{C}(4)-\mathrm{O}(2)-\mathrm{C}(5)$ & $-24.3(5)$ \\
\hline $\mathrm{O}(3)-\mathrm{C}(5)-\mathrm{O}(4)-\mathrm{C}(6)$ & $176.3(3)$ \\
\hline $\mathrm{O}(2)-\mathrm{C}(5)-\mathrm{O}(4)-\mathrm{C}(6)$ & $-3.5(5)$ \\
\hline $\mathrm{C}(11)-\mathrm{C}(6)-\mathrm{O}(4)-\mathrm{C}(5)$ & $-91.2(4)$ \\
\hline $\mathrm{C}(3)-\mathrm{C}(6)-\mathrm{O}(4)-\mathrm{C}(5)$ & $32.2(4)$ \\
\hline $\mathrm{C}(7)-\mathrm{C}(6)-\mathrm{O}(4)-\mathrm{C}(5)$ & $149.8(3)$ \\
\hline
\end{tabular}

Symmetry transformations used to generate equivalent atoms: 
Thermal ellipsoid diagram for 19:

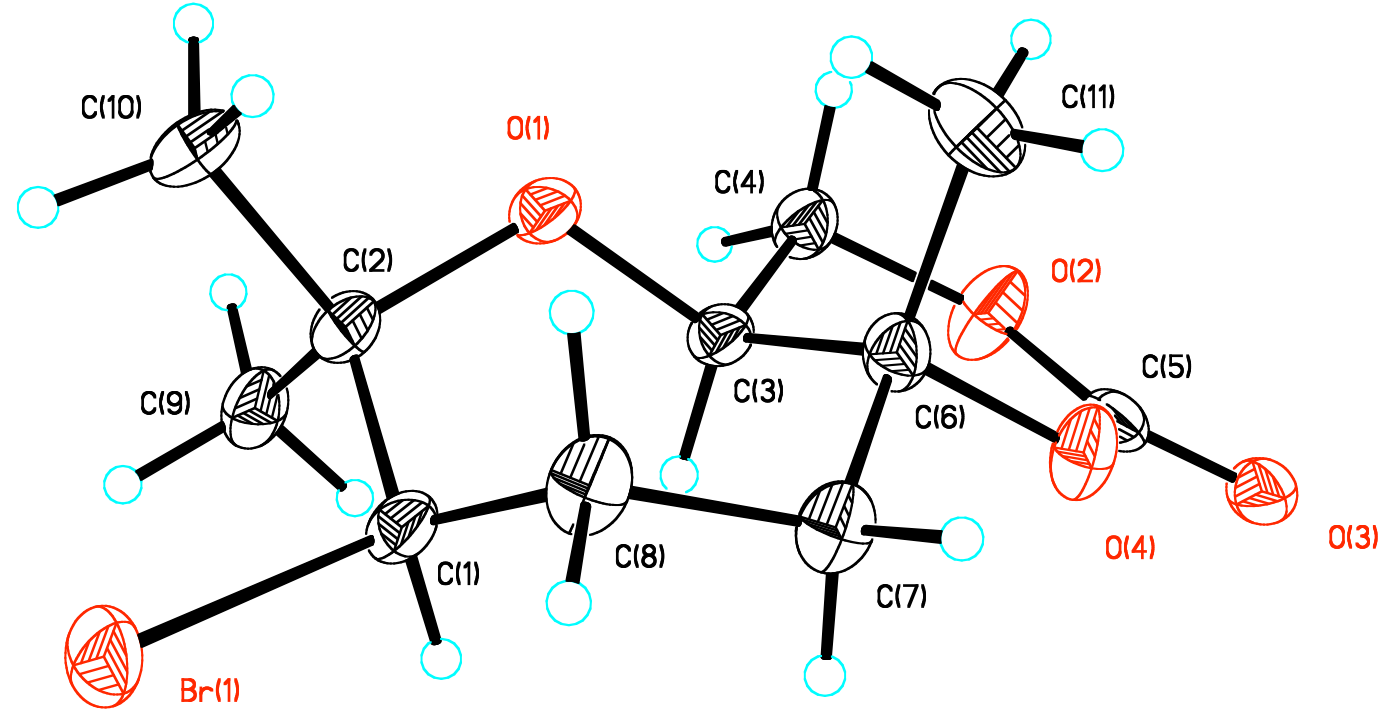

